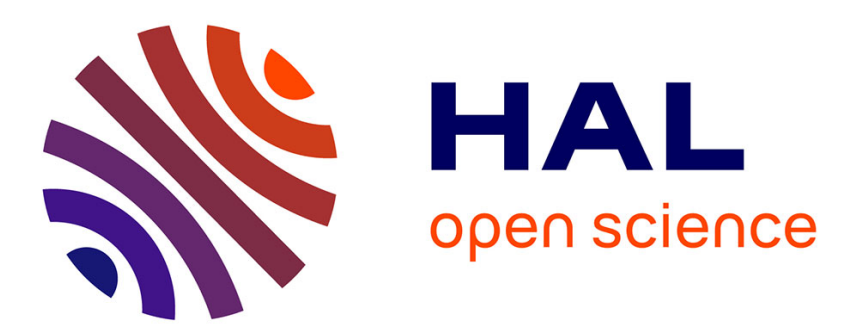

\title{
Proto-neutron star evolution with improved charged-current neutrino-nucleon interactions
}

A Pascal, J Novak, M Oertel

\section{To cite this version:}

A Pascal, J Novak, M Oertel. Proto-neutron star evolution with improved charged-current neutrinonucleon interactions. Monthly Notices of the Royal Astronomical Society, In press, 10.1093/mnras/stac016 . hal-03512018

\section{HAL Id: hal-03512018 \\ https://hal.science/hal-03512018}

Submitted on 5 Jan 2022

HAL is a multi-disciplinary open access archive for the deposit and dissemination of scientific research documents, whether they are published or not. The documents may come from teaching and research institutions in France or abroad, or from public or private research centers.
L'archive ouverte pluridisciplinaire HAL, est destinée au dépôt et à la diffusion de documents scientifiques de niveau recherche, publiés ou non, émanant des établissements d'enseignement et de recherche français ou étrangers, des laboratoires publics ou privés. 


\title{
Proto-neutron star evolution with improved charged-current neutrino-nucleon interactions
}

\author{
A. Pascal, ${ }^{1 \star}{ }^{\prime}$. Novak ${ }^{1} \dagger$ and M. Oertel ${ }^{1} \ddagger$ \\ ${ }^{1}$ Laboratoire Univers et Théories, Observatoire de Paris, Université PSL, CNRS, Université de Paris, 92190 Meudon, France
}

5 January 2022

\begin{abstract}
We perform simulations of the Kelvin-Helmholtz cooling phase of proto-neutron stars with a new numerical code in spherical symmetry and using the quasi-static approximation. We use for the first time the full set of charged-current neutrino-nucleon reactions, including neutron decay and modified Urca processes, together with the energy-dependent numerical representation for the inclusion of nuclear correlations with random-phase approximation. Moreover, convective motions are taken into account within the mixing-length theory. As we vary the assumptions for computing neutrino-nucleon reaction rates, we show that the dominant effect on the cooling timescale, neutrino signal and composition of the neutrino-driven wind comes from the inclusion of convective motion. Computation of nuclear correlations within the random phase approximation, as compared to mean field approach, has a relatively small impact.
\end{abstract}

Key words: stars:neutron - neutrinos - methods: numerical

\section{INTRODUCTION}

Neutrons stars are formed during the core-collapse of massive stars at the end of their evolution. During this collapse the core reaches supra-nuclear densities and bounces, generating a shock that can trigger a supernova explosion. The central object left after the bounce is called a proto-neutron star (PNS). The PNS has a much larger radius and is still an extremely hot and lepton rich object compared with cold neutron stars in weak ( $\beta$-)equilibrium. During its evolution, this object will accrete infalling material and slowly deleptonize, contract and cool down by the Kelvin-Helmholtz mechanism to become either a stellar black hole or a neutron star (see e.g. Prakash et al. 2001).

In the last few decades the sensitivity of neutrino detectors has greatly improved, and a detailed modelling of the emission of neutrinos associated to core-collapse and subsequent PNS evolution is needed in order to interpret the data that shall be obtained from the next galactic supernova (see e.g. Nakazato et al. 2021). This could lead to a better understanding of the central engine of core-collapse supernovae $(\mathrm{CCSN})$ and of supernova nucleosynthesis. In addition, thermodynamic conditions, i.e. temperatures and densities, in PNS are similar to hypermassive neutron stars formed during mergers of neutron star binaries (see e.g. Paschalidis et al. 2012; Iosif \& Stergioulas 2021), therefore the study of PNS evolution can lead to a better understanding of matter prop-

\footnotetext{
* E-mail: aurelien.pascal@obspm.fr

$\dagger$ E-mail: jerome.novak@obspm.fr

† E-mail: micaela.oertel@obspm.fr
}

erties and neutrino interactions involved in binary mergers, too.

Long-term multi-dimensional CCSN simulations are, however, still beyond our reach due to the very different timescales involved: typical hydrodynamic timescales are orders of magnitude below the typical deleptonization and cooling time. Therefore we have to make some compromise to study late-time CCSN and PNS evolution. Work on this subject exists for more than 25 years. There are two types of approaches to tackle this problem:

(i) stick with $1 \mathrm{D}$ CCSN simulations and either artificially enhance the neutrino heating to trigger the explosion (see e.g. Fischer et al. 2010), use a low mass O-Ne-Mg core, which are known to explode even in 1D simulations (see e.g. Hüdepohl et al. 2010), or switch from multi-D to $1 \mathrm{D}$ by angle averaging after the explosion (see e.g. Suwa 2014)

(ii) use the fact that the PNS has a very long evolution timescale compared with the neutrino emission and use a quasi-static model of its Kelvin-Helmholtz contraction. There are numerous works which have used this method, among others we can mention Burrows \& Lattimer (1986); Keil \& Janka (1995); Sumiyoshi et al. (1995); Pons et al. (1999) and Roberts (2012). We have chosen this kind of approach within our work.

Although state-of-the-art multi-dimensional simulations are still bound to about $1 \mathrm{~s}$ of evolution, they are very useful for calibrating simpler models describing evolution at longer timescales. In particular, they tend to show that the convective motions in the PNS are extremely significant and change quantitatively the behaviour of the PNS, see e.g. Nagakura et al. (2020). A very simple method to implement convective 
effects in quasi-static models of PNS is the use of the mixing length theory (MLT), see e.g. Roberts et al. (2012b) and Mirizzi et al. (2016). This method does not cover all features of convection, for instance convective overshooting cannot be described, but it mimics in a computationally very efficient way the main effects of convection. We have therefore adopted the MLT scheme to include convective effects in our work.

Obviously, PNS evolution and the resulting neutrino emission is not only sensitive to hydrodynamics and matter properties via an equation of state (EoS), but the interaction of neutrinos with the dense and hot matter is an extremely important ingredient, too, see e.g. the discussions in Pons et al. (1999); Roberts et al. (2012a); Martínez-Pinedo et al. (2012). Since the different simulations are computationally expensive, many authors prefer to employ analytical expressions for the corresponding interaction rates which imply often very crude approximations. Here, we will focus the discussion on charged-current neutrino-nucleon interactions and use standard rates for all other processes. Analytic expressions for the former can be obtained in limiting cases: assuming noninteracting nucleons for computing the matrix element and either neglecting the momentum transfer between the nucleons (Bruenn 1985) or taking the nucleon momenta on the Fermi surface (Yakovlev et al. 2001). The former is valid at low densities $\lesssim 10^{-4} \mathrm{fm}^{-3}$, conditions typically found in the early CCSN neutrinosphere, and the latter in degenerate matter, typically for the cooling of older neutron stars with temperatures $\ll 1 \mathrm{MeV}$. During PNS evolution, the neutrinosphere moves inwards to higher densities and temperatures vary between a few tens of $\mathrm{MeV}$ in the early times and a few $\mathrm{MeV}$ at the later stages. Thus matter in the relevant regions is neither degenerate nor at sufficiently low densities and corrections to the above analytical expressions have to be considered.

In this context, Roberts et al. (2012a) and Martínez-Pinedo et al. (2012) have pointed out the importance of mean field corrections for nucleon masses and chemical potentials included in a way consistent with the underlying EoS for PNS evolution. Roberts et al. (2012a,b) additionally include the full phase space integration (Reddy et al. 1998), i.e. consider arbitrary momentum transfer between the nucleons. In Fischer et al. (2020a) it is shown that taking into account inverse neutron decay in addition to the standard electron and positron capture reactions (and their inverse) influences in particular the opacity of low energy electron antineutrinos and consequently the composition of the neutrino-driven wind. In addition, several authors have pointed out since decades that in dense matter nuclear correlations beyond mean field can considerably modify the neutrino opacities: Burrows \& Sawyer (1998, 1999); Reddy et al. (1999); Navarro et al. (1999); Margueron et al. (2004); Horowitz \& Schwenk (2006); Horowitz et al. (2017). Nuclear correlations treated within the "Random Phase Approximation" (RPA) have been considered for PNS evolution by Pons et al. (1999); Roberts et al. (2012b); Roberts \& Reddy (2017) suggesting a potential influence on the neutrino spectra after several seconds of evolution when matter starts to become transparent to neutrinos. However, following Reddy et al. (1999) only grey, i.e. (anti-)neutrino energy independent, correction factors to the mean field expressions have been implemented. As discussed in Oertel et al. (2020), the importance of RPA correlations is energy dependent and a density and temperature dependent shift in reaction thresholds is induced. The full physics can thus not be included into a grey factor.

Some of these improvements are modifying the analytical rates by several orders of magnitude, such that it became a priority to perform CCSN and PNS evolution simulations with state of the art neutrino interactions in order to test them against previous simulations and predict the emitted neutrino signal. In Oertel et al. (2020) a first step has been made in this direction, electron (anti)-neutrino opacities from charged current neutrino nucleon interactions are computed including mean field corrections, optionally RPA correlations and include the full phase space integration. As reactions, electron and positron capture, neutron and proton decay as well as their inverse processes have been considered. An interpolation scheme has been developed which allows to provide the obtained opacity data with the full dependence on neutrino energy $E_{\nu}$, baryon number density $n_{B}$, temperature $T$ and electron fraction $Y_{e}$, with tables of interpolation coefficients publicly available via the COMPOSE data base $^{1}$ (Typel et al. 2015). The feasibility of CCSN simulations employing these opacities with only a minor excess in computation time compared with analytical rates has been demonstrated in Oertel et al. (2020). In the present work we perform a step further by using those rates in a simulation of PNS evolution. Please note that at later times of PNS evolution reactions can become important which are usually not included in the simulations. In particular, it should be noted that neutron decay is one of the main reactions establishing weak equilibrium in cold stars as well as the so-called modified Urca processes, depending on the kinematic conditions. Here, we will thus study the impact of RPA correlations with the full energy dependence and the role of different reactions for the PNS evolution and the transition to neutrino transparency. We also provide for the first time simulations with the most complete set of neutrino charged-current reactions and at the same time an effective model for convection. This enables us to compare quantitatively the different effects potentially influencing the PNS evolution.

This paper is organised as follows: in Section 2 we present the code and the numerical model used in our simulations and Section 3 is focused on the presentation of the various prescriptions for the computation of charged current reaction rates. The results of our study are discussed in Section 4, we start with a comparison between models including convective motions in PNS evolution using MLT and models without MLT in Section 4.1 and we then discuss the impact of the different charged-current neutrino nucleon interactions in Section 4.2. Our conclusions are presented in Section 5.

\section{A CODE FOR QUASI-STATIC PROTO-NEUTRON STAR EVOLUTION IN SPHERICAL SYMMETRY}

This section is devoted to the presentation of the code used in our PNS simulations. It is based on a quasi-static approach with a Lagrangian grid and a stationary neutrino transport scheme. Evolution of entropy and lepton number (Sec. 2.3) is obtained through neutrino transport (Sec. 2.4), and at each

\footnotetext{
1 https://compose.obspm.fr/
} 
time-step equations for hydrostatic equilibrium are solved (Sec. 2.2), assuming thus that the hydrodynamics time scale is much shorter than the neutrino cooling one.

\subsection{Initial models}

Starting with the progenitor model s15 from Woosley et al. (2002), we run the spherically-symmetric version of the CCSN code CoCoNuT (Dimmelmeier et al. 2005), using the "Fast Multigroup Transport" (FMT) scheme for neutrino transport (Müller \& Janka 2015), with the same method as in Oertel et al. (2020). Each model has been computed such that the EoS and the neutrino interactions are consistent with the PNS cooling simulation. To emulate the departure of the shock and obtain an isolated PNS, we simply discard all the matter behind the stalled shock, at about $500 \mathrm{~ms}$ after bounce. We thus obtain a PNS with a baryon mass of $M_{B}=1.6 M_{\odot}$.

\subsection{Stellar structure}

From the quasi-static assumption, gravitational field is static and therefore we consider a static, spherically-symmetric spacetime. The metric is written in Schwarzschild gauge, so that we obtain the usual Tolman-Oppenheimer-Volkoff (TOV) set of equations for the hydrostatic equilibrium:

$d s^{2}=-\alpha^{2}(r) c^{2} d t^{2}+\psi^{2}(r) d r^{2}+r^{2}\left(d \theta^{2}+\sin ^{2} \theta d \varphi^{2}\right)$

where $c$ is the speed of light in vacuum, $\alpha$ is called the lapse function (from $3+1$ formalism) and $\psi$ is a radial metric potential. The system of coordinates is such that $t$ is the time measured at infinity, $r$ is the areal radius, and $\theta$ and $\varphi$ are standard angular coordinates. The metric is computed by solving the TOV equations, assuming that the star is composed of a perfect fluid :

$$
\begin{aligned}
\frac{1}{\psi} & =\sqrt{1-\frac{2 G m}{r c^{2}}} \\
\frac{d m}{d r} & =4 \pi r^{2} \frac{\mathcal{E}}{c^{2}} \\
g(r) & =c^{2} \frac{d \ln \alpha}{d r}=\psi^{2} G\left(\frac{m}{r^{2}}+4 \pi r \frac{P}{c^{2}}\right),
\end{aligned}
$$

where $G$ is the gravitational constant, $m$ is a metric potential that is equal to the system's ADM mass when taken at the star's surface, $g$ is the local gravitational acceleration, $\mathcal{E}$ the fluid energy density and $P$ its pressure. The hydrostatic equilibrium equation is given by

$\frac{d P}{d r}=-(\mathcal{E}+P) g(r)$.

The boundary conditions are given by $m(0)=0, \alpha(R) \psi(R)=$ 1 and $P(R)=P_{s}$ where $R$ is the radius of the star and $P_{s}$ is a surface pressure, whose value is chosen to have a negligible effect on the solution while ensuring the numerical stability of the algorithm. In the simulations presented in this work we took $P_{s}=10^{-6} \mathrm{MeV} \mathrm{fm}^{-3}$.

As we consider a Lagrangian evolution scheme, the radial coordinate $r$ is an unknown, too, and an additional equation is solved together with the previous system :

$\frac{d r}{d a}=\frac{1}{4 \pi r^{2} n_{B} \psi}$, where $a$ is the enclosed baryon number (fixed during the evolution and used as a Lagrangian coordinate), and $n_{B}$ is the baryon number density.

Finally, in order to close the system of equations, we need an EoS relating thermodynamic variables. In our case it shall be a 3-parameter one, depending namely on temperature $T$, baryon density $n_{B}$ and electron fraction $Y_{e}$. In this work we have used three different EoS models: (i) the nuclear statistical equilibrium (NSE) model by Gulminelli \& Raduta (2015) ("RG(SLy4)"), employing the SLy4 effective interaction for nucleons (Chabanat et al. 1997); (ii) the NSE model by Hempel \& Schaffner-Bielich (2010), employing the DD2 effective interaction for nucleons (Typel et al. 2010) ("HS(DD2)"); (iii) the "SRO(APR)" model (Constantinou et al. 2014; Schneider et al. 2019). The latter is based on the APR EoS (Akmal et al. 1998), which itself is partly adjusted to the variational calculation of Akmal \& Pandharipande (1997). For all three models. EoS data have been obtained from the CompOSE database (Typel et al. 2015).

\subsection{Evolution equations}

The time evolution from one quasi-static configuration (computed using the TOV system given above) to the next is done by considering the effects of neutrino interactions on the stellar matter via lepton number and energy conservation. These are given by

$\begin{aligned} \nabla_{\mu}\left(u^{\mu} n_{B} Y_{e}\right) & =\Gamma_{\bar{\nu}_{e}}-\Gamma_{\nu_{e}} \\ u_{\nu} \nabla_{\mu}\left(T^{\mu \nu}\right) & =-\left(Q_{\nu_{e}}+Q_{\bar{\nu}_{e}}+4 Q_{\nu_{x}}\right),\end{aligned}$

where $u^{\mu}$ is the fluid four-velocity, $T^{\mu \nu}$ the fluid's energymomentum tensor, $\Gamma_{\nu}$ the neutrino production rate per volume unit, and $Q_{\nu}$ the neutrino heat function for each neutrino or anti-neutrino flavor $\nu_{i}$. For a perfect fluid these equations can easily be recast as

$$
\begin{aligned}
u^{\mu} \nabla_{\mu}\left(Y_{e}\right) & =\frac{1}{\alpha} \frac{D Y_{e}}{D t}=\frac{\Gamma_{\bar{\nu}_{e}}-\Gamma_{\nu_{e}}}{n_{B}} \\
u^{\mu} \nabla_{\mu}(s) & =\frac{1}{\alpha} \frac{D s}{D t}=\frac{Q_{\nu_{e}}+Q_{\bar{\nu}_{e}}+4 Q_{\nu_{x}}}{n_{B} T}-\frac{\mu_{e}\left(\Gamma_{\bar{\nu}_{e}}-\Gamma_{\nu_{e}}\right)}{n_{B} T}
\end{aligned}
$$

where $s$ is the entropy per baryon, $\mu_{e}$ the electron chemical potential and $D / D t$ is the Lagrangian derivative. Both source terms, $\Gamma_{\nu}$ and $Q_{\nu}$, are obtained from a neutrino transport scheme, by using Eqs. (12) and (13), see the Sec. 2.4 below.

\subsection{Neutrino transport}

In order to model neutrino transport, we use the FMT scheme (see Müller \& Janka 2015), which relies on a stationary approximation of the transport equation,

$p^{i} \frac{\partial f}{\partial x^{i}}-\Gamma_{\mu \nu}^{i} p^{\mu} p^{\nu} \frac{\partial f}{\partial p^{i}}=u_{\mu} p^{\mu} \mathcal{B}[f]$.

$f$ denotes here the neutrino distribution function, $p^{\mu}$ the neutrino four-momentum and $\mathcal{B}[f]$ the collision integral computed in the fluid rest frame.

The solution at high optical depth is obtained with a twostream approximation and the solution at low optical depth is obtained with a two-moment closure. The procedure is detailed in appendix A, whereas the treatment of the collision integral is detailed in Section 3 for charged current 
reactions on nucleons and in appendix B for all other processes. It should be stressed in this context that we model neutrino-nucleon scattering with full inelastic rates, as given by Thompson et al. (2000).

In this kind of stationary approximation, the source terms are obtained via the divergence of neutrino fluxes:

$$
\begin{aligned}
\Gamma_{\nu} & =\frac{1}{r^{2} \psi \alpha} \frac{d}{d r}\left(r^{2} \alpha F_{\nu, n}\right) \\
Q_{\nu} & =\frac{1}{r^{2} \psi \alpha^{2}} \frac{d}{d r}\left(r^{2} \alpha^{2} F_{\nu, e}\right),
\end{aligned}
$$

where $F_{\nu, n}$ is the outgoing number flux of neutrino $\nu$ and $F_{\nu, e}$ is the outgoing energy flux carried by neutrinos $\nu$. The total luminosities are then given by

$$
\begin{aligned}
L_{\nu, n} & =4 \pi R^{2} \alpha(R) F_{\nu, n}(R) \\
L_{\nu, e} & =4 \pi R^{2} \alpha(R)^{2} F_{\nu, e}(R),
\end{aligned}
$$

where $L_{\nu, n}$ is the neutrino number luminosity (in $\mathrm{s}^{-1}$ ) and $L_{\nu, e}$ is the energy luminosity (in $\mathrm{erg} \mathrm{s}^{-1}$ ).

\subsection{Convection using the mixing length theory}

We study the effect of convection on PNS evolution within the MLT, which models convection in spherical symmetry as a diffusive effect occurring in zones with unstable stratification. The stability criterion for the stratified structure of a PNS is given by the Ledoux criterion (see e.g. Roberts et al. 2012b):

$C_{L}(r)=\frac{1}{\Gamma_{n_{B}}}\left(\Gamma_{s} \frac{\partial \ln s}{\partial r}+\Gamma_{Y_{e}} \frac{\partial \ln Y_{e}}{\partial r}\right) \geq 0$,

where $r$ is the radial coordinate and $\Gamma_{n_{B}}=\left(\frac{d \ln P}{d \ln n_{B}}\right)_{s, Y_{e}}$, $\Gamma_{s}=\left(\frac{d \ln P}{d \ln s}\right)_{n_{B}, Y_{e}}, \Gamma_{Y_{e}}=\left(\frac{d \ln P}{d \ln Y_{e}}\right)_{n_{B}, s}$.

The quantity $C_{L}(r) d r$ can be interpreted as the relative variation of density $\Delta n_{B} / n_{B}$ occurring during the small vertical adiabatic displacement of a mass element over a distance $d r$. Several authors (e.g. Epstein 1979; Keil et al. 1996; Roberts et al. 2012b) have argued that, because the neutrinos are trapped and in equilibrium with the fluid in large regions of the PNS, one should consider the lepton fraction instead of the electron fraction to compute the Ledoux criterion. We think however, that as convection is a purely hydrodynamic feature appearing when considering multi-dimensional versions of Eqs. (7)-(8), one should use only electron fraction, to be consistent with the hydrodynamic model.

The distinction is then made between

- areas where $C_{L}(r)>0$, where buoyancy acts as a restoring force. They can be subject to gravity waves ;

- areas where $C_{L}(r)<0$, which are unstable and can be subject to convective motion ;

- areas where $C_{L}(r)=0$, which are in a state of neutral buoyancy. Convective motions tend to bring unstable areas towards this state.

In the MLT, the convective motion in Ledoux-unstable areas is modelled with diffusion equations for the entropy and the electron number,

$$
\begin{aligned}
\frac{1}{\alpha} \frac{D Y_{e}}{D t} & =\frac{1}{r^{2} \alpha \psi} \frac{\partial}{\partial r}\left(\alpha r^{2} D^{\mathrm{MLT}} n_{B} \frac{\partial Y_{e}}{\partial r}\right) \\
\frac{1}{\alpha} \frac{D s}{D t} & =\frac{1}{r^{2} \alpha \psi} \frac{\partial}{\partial r}\left(\alpha r^{2} D^{\mathrm{MLT}} n_{B} \frac{\partial s}{\partial r}\right)
\end{aligned}
$$

where $D^{\text {MLT }}$ is the MLT diffusion coefficient. We estimate it by using the same procedure as in Mirizzi et al. (2016): we have $D^{\mathrm{MLT}}=v_{\mathrm{c}} \lambda_{P}$, where $v_{\mathrm{c}}$ is the convection velocity and $\lambda_{P}$ is the length scale over which convective turnover occurs, or the so-called mixing length. This length scale is assumed to be of the same order of magnitude as that of pressure variation,

$\lambda_{P}=\xi\left(\frac{\partial \ln P}{\partial r}\right)^{-1}$

where $\xi$ is a coefficient of order unity. The exact value of $\xi$ does not have a strong influence on the results, and we use $\xi=1$ as the standard value. We have checked that varying its value by $\pm 20 \%$ does not change the results of our simulations within the overall accuracy of the model.

The convection velocity $v_{\mathrm{c}}$ is estimated using energy conservation during a vertical displacement of $\lambda_{P}$ :

$v_{\mathrm{c}}= \begin{cases}\lambda_{P} \sqrt{2 g\left|C_{L}\right|} & \text { if } C_{L}(r) \leq 0 \\ 0 & \text { if } C_{L}(r) \geq 0,\end{cases}$

where $g$ is the local gravitational acceleration introduced in Eq. (4).

The PNS evolution equations are then solved with a semiimplicit scheme: the neutrino part (namely Eqs. (9) and (10)) is solved with an explicit scheme whereas the MLT part (Eqs. (15) and (16)) is solved implicitly. The timestep is limited by the relative change in $s$ and $Y_{e}$ induced by the neutrino sources. The explicit integration scheme for the neutrino part has the advantage of allowing to perform larger and larger timesteps as the neutrino emissivity decreases while not having to deal with the heavy computational cost of an implicit scheme for neutrino transport.

\section{TREATMENT OF CHARGED CURRENT INTERACTIONS WITH NUCLEONS}

In this section we present the treatment of charged current neutrino-nucleon interactions. For such processes the collision integral in the neutrino transport equation (11) is linear in $f_{\nu}$ and can be written in the form:

$\mathcal{B}[f]=j(1-f)-\frac{1}{\lambda} f=\kappa^{*}\left(f^{(e q)}-f\right)$,

where $j$ is the emissivity, $\lambda$ the absorption mean free path and $\kappa^{*}=j+\frac{1}{\lambda}$ is the opacity corrected for stimulated absorption. It should be stressed that in our work we consider only charged-current processes involving electrons, and we neglect the effect of muonic processes. Such processes have long been thought to have a negligible influence on CCSN because of the relatively low abundance of muons compared with electrons due to their much higher mass. The recent work of Bollig et al. (2017) has, however, shown that the appearance of muons has a significant effect on the CCSN evolution by softening the EoS and muonic processes have gained interest. 
Thus, the recent study by Fischer et al. (2020b) has quantified the effect of muonic charged-current processes on the neutrino luminosities. We will consider the impact of muonic processes on PNS evolution in a future work.

\subsection{Direct Urca processes}

Direct Urca processes are the dominant and simplest charged current neutrino-nucleon interactions involving electron neutrinos and antineutrinos. The processes for electron neutrinos are

$p+e^{-} \leftrightarrows n+\nu_{e} \quad p \leftrightarrows n+e^{+}+\nu_{e}$

and the corresponding processes for antineutrinos are

$n+e^{+} \leftrightarrows p+\bar{\nu}_{e} \quad n \leftrightarrows p+e^{-}+\bar{\nu}_{e}$

In this work we employ three different approximations for computing the rates for the above processes. The first one is the elastic approximation ("elastic MF") in which the momentum transfer between the nucleons is neglected (see Bruenn 1985). Mean field effects are added as effective masses and single particle potentials for the nucleons (Reddy et al. 1998). In addition we use the Mean Field ("MF") approximation with full phase space integration, and a scheme including nuclear correlations within the Random Phase Approximation $(R P A)$. In particular, we used the so-called "RPA $t_{3}^{\prime}$ " approximation, which considers an additional repulsive term in the residual interaction in the axial channel to prevent instabilities at high densities. For details about the different approximation schemes and the practical implementation, we refer to Oertel et al. (2020). In particular, "MF" and "RPA $t_{3}^{\prime}$ " rates are provided via tabulated interpolation coefficients for fully energy dependent precomputed opacities in order to avoid the heavy additional computational cost of the integration of opacities with full space or in RPA.

The elastic approximation is still very commonly used in many recent simulations of PNS evolution (see e.g. Li et al. 2021; Nakazato \& Suzuki 2020), as it is quite accurate at high temperatures and neutrino energies and relatively low densities, i.e. for the typical conditions close to the neutrinosphere in CCSN. We should, however, point out again that the elastic approximation looses accuracy during PNS evolution with the neutrinosphere moving to higher densities and the subsequent cooling of the PNS. At higher densities, the elastic rates can differ from the full ones by orders of magnitude and in addition, $\beta$-equilibrium is displaced towards more neutron rich conditions. Both effects are illustrated in Fig. 1, where electron neutrino and antineutrino production rates are shown as functions of the electron fraction for two different temperatures and a baryon number density of $n_{B}=0.1 \mathrm{fm}^{-3}$, employing either the elastic approximation or the full phase space integration, including mean field effects in both cases. For the shown conditions, matter can be considered as transparent to (anti)-neutrinos and $\beta$-equilibrium is obtained if neutrino and antineutrino rates are equal, i.e. at the intersections of the $\nu_{e}$ and $\bar{\nu}_{e}$ curves. It is obvious that with the elastic rates, $\beta$-equilibrium occurs at lower values of $Y_{e}$.

It can be seen in addition that, as noticed previously by Alford \& Harris (2018); Alford et al. (2021), $\beta$-equilibrium in neutrino transparent matter at non zero temperature does not occur at the point where chemical potentials fulfil the usual $\beta$-equilibrium condition for cold neutron stars $\left(\mu_{n}=\mu_{p}+\mu_{e}\right.$ which stems from the Fermi surface approximation, indicated by the red dashed line in Fig. 1) but depends explicitly on the neutrino production rates instead. Let us stress that, following Fig. 1, only the rates including full kinematics allow to recover the Fermi surface approximation at low temperatures and thus the correct $\beta$-equilibrium conditions upon evolving from a PNS to a neutron star.

\subsection{Modified Urca processes}

From neutron star cooling, it is well known that under degenerate conditions in cold neutron star matter the direct Urca processes, see Eq. (20), are kinematically forbidden unless the proton fraction exceeds roughly $10 \%$. In the same way, during the late stages of PNS evolution they can become strongly suppressed for some neutrino energies. In this case, the so-called modified Urca (mUrca) processes become relevant, see also the discussion of $\beta$-equilibrium in the hot merger remnant in Alford \& Harris (2018) and Alford et al. (2021). They involve a spectator nucleon $N$ allowing to lift the kinematic restrictions of the direct processes :

$$
\begin{array}{ll}
p+e^{-}+N \leftrightarrows n+\nu_{e}+N & p+N \leftrightarrows n+e^{+}+\nu_{e}+N \\
n+e^{+}+N \leftrightarrows p+\bar{\nu}_{e}+N & n+N \leftrightarrows p+e^{-}+\bar{\nu}_{e}+N
\end{array}
$$

The relevant reactions rates have been studied extensively for degenerate conditions in cold neutron stars following the seminal work by Friman \& Maxwell (1979), whereas much less effort has been dedicated to hot matter. Due to their importance for CCSN neutrino spectra, the corresponding neutral current reaction rates have, however, been studied in more detail (see e.g. Hannestad \& Raffelt 1998). Here we will follow the phenomenological approach of Roberts et al. (2012a), who have adapted the general framework for neutral current reactions in Lykasov et al. (2008) to the above charged current reactions.

The idea is that the excitation of two-particle states, as required to describe the reactions in Eq. (22), leads to a collisional broadening which can be incorporated as finite quasiparticle lifetime $\tau$ in the nuclear response function entering the rate calculation (see Roberts et al. 2012a, for details). Because of vector current conservation, the vector current contribution to the neutral current rate vanishes in the limit of zero-momentum transfer, so that it is generally assumed that the axial current contribution dominates. In order to take mUrca reactions into account, we have therefore implemented a finite width for the quasi-particles only in the axial channel with numerical values for the lifetime taken from Bacca et al. (2012), see appendix C for more details.

Let us stress that modelling mUrca processes in this way should not be considered as quantitatively reliable. Among others, the momentum transfer for the charged-current processes in dense matter is not negligible, so that the contribution of the vector channel merits further investigation. The values for the quasi-particle lifetime in Bacca et al. (2012) have been obtained in the limit of vanishing momentum transfer and for neutron matter for some selected temperatures and baryon number densities. They can thus only be 


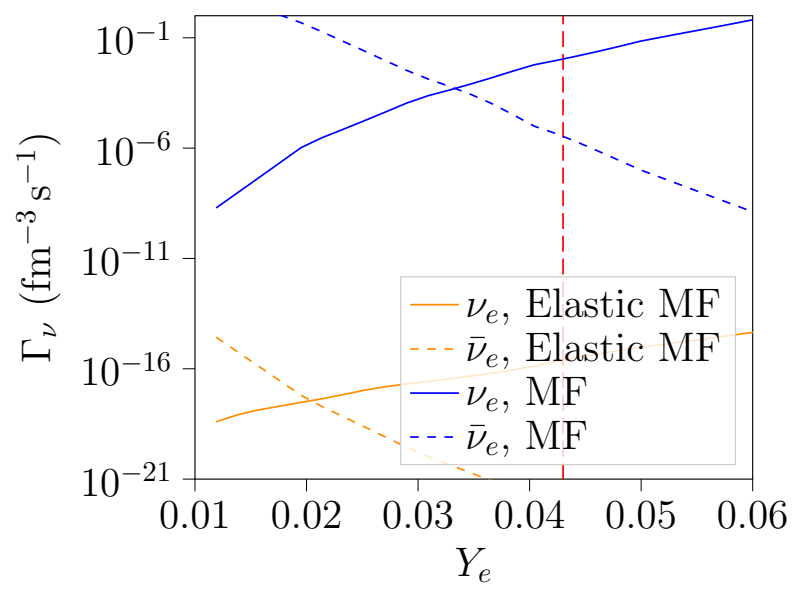

(a) $\mathrm{T}=2 \mathrm{MeV}$

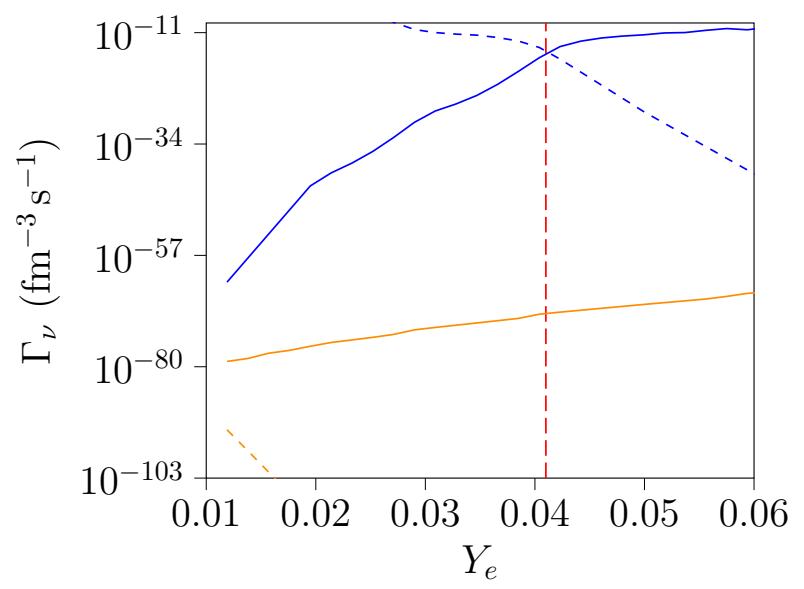

(b) $T=0.3 \mathrm{MeV}$

Figure 1. Electron neutrino and antineutrino production rates per unit volume, at the density $n_{B}=0.1 \mathrm{fm}^{-3}$, for two different temperatures as functions of the electron fraction. Both temperature values are low enough for matter to be considered as transparent. The RG(SLy4) EoS has been used. $\beta$-equilibrium is obtained if neutrino and antineutrino rates are equal, i.e. at the intersections of the $\nu_{e}$ and $\bar{\nu}_{e}$ curves. The vertical red dashed line represents the equilibrium electron fraction predicted by the Fermi surface approximation.

\begin{tabular}{|c|c|c|c|c|c|c|c|c|c|c|c|}
\hline $\begin{array}{c}t \\
(\mathrm{~s})\end{array}$ & $\begin{array}{c}T \\
(\mathrm{MeV}) \\
\end{array}$ & $\begin{array}{c}n_{B} \\
\left(\mathrm{fm}^{-3}\right) \\
\end{array}$ & $Y_{e}$ & EoS & $x_{n}$ & $x_{p}$ & $\begin{array}{c}U_{n} \\
(\mathrm{MeV}) \\
\end{array}$ & $\begin{array}{c}U_{p} \\
(\mathrm{MeV}) \\
\end{array}$ & $\begin{array}{c}\Delta U \\
(\mathrm{MeV})\end{array}$ & $\begin{array}{c}m_{n}^{*} \\
(\mathrm{MeV})\end{array}$ & $\begin{array}{c}m_{p}^{*} \\
(\mathrm{MeV}) \\
\end{array}$ \\
\hline \multirow[t]{4}{*}{0} & 15.0 & 0.250 & 0.30 & RG(SLy4) & 0.704 & 0.296 & 345.8 & 258.7 & 6.03 & 519.7 & 599.5 \\
\hline & 26.9 & 0.017 & 0.22 & $\mathrm{RG}(\mathrm{SLy} 4)$ & 0.702 & 0.155 & 39.05 & 7.586 & 12.16 & 893.7 & 911.7 \\
\hline & 11.2 & $6.58 \cdot 10^{-3}$ & 0.12 & $\mathrm{RG}(\mathrm{SLy} 4)$ & 0.751 & 0.051 & 16.24 & -1.079 & 7.30 & 921.1 & 929.9 \\
\hline & 4.46 & $9.35 \cdot 10^{-6}$ & 0.34 & RG(SLy4) & 0.655 & 0.339 & $5.772 \cdot 10^{-3}$ & $-8.918 \cdot 10^{-3}$ & $8.025 \cdot 10^{-3}$ & 939.5 & 938.3 \\
\hline \multirow[t]{4}{*}{0.7} & 20.1 & 0.319 & 0.29 & RG(SLy4) & 0.710 & 0.289 & 399.9 & 321.9 & -8.237 & 462.2 & 547.1 \\
\hline & 35.7 & 0.083 & 0.12 & $\mathrm{RG}(\mathrm{SLy} 4)$ & 0.868 & 0.108 & 188.7 & 54.25 & 39.57 & 723.5 & 817.2 \\
\hline & 18.8 & 0.033 & 0.12 & $\mathrm{RG}(\mathrm{SLy} 4)$ & 0.751 & 0.055 & 75.20 & 6.480 & 24.31 & 854.3 & 897.4 \\
\hline & 4.18 & $7.86 \cdot 10^{-4}$ & 0.06 & $\mathrm{RG}(\mathrm{SLy} 4)$ & 0.848 & 0.015 & 2.229 & -0.615 & 1.384 & 937.1 & 937.3 \\
\hline \multirow[t]{4}{*}{5.1} & 48.6 & 0.439 & 0.07 & RG(SLy4) & 0.931 & 0.069 & 475.9 & 375.4 & -80.93 & 353.5 & 533.6 \\
\hline & 27.6 & 0.256 & 0.07 & $\mathrm{RG}(\mathrm{SLy} 4)$ & 0.931 & 0.069 & 379.3 & 193.8 & 11.48 & 477.8 & 650.6 \\
\hline & 16.3 & 0.150 & 0.07 & $\mathrm{RG}(\mathrm{SLy} 4)$ & 0.932 & 0.068 & 284.1 & 97.58 & 39.46 & 599.7 & 745.5 \\
\hline & 3.54 & $8.58 \cdot 10^{-3}$ & 0.06 & RG(SLy4) & 0.637 & $5.01 \cdot 10^{-5}$ & 17.82 & -2.541 & 8.494 & 919.7 & 930.3 \\
\hline \multirow[t]{4}{*}{13.1} & 9.70 & 0.514 & 0.06 & RG(SLy4) & 0.939 & 0.061 & 487.4 & 443.8 & -138.9 & 319.3 & 500.5 \\
\hline & 5.71 & 0.314 & 0.06 & $\mathrm{RG}(\mathrm{SLy} 4)$ & 0.941 & 0.059 & 407.8 & 239.8 & -16.09 & 429.2 & 612.0 \\
\hline & 3.75 & 0.196 & 0.05 & $\mathrm{RG}(\mathrm{SLy} 4)$ & 0.946 & 0.054 & 329.0 & 130.8 & 29.96 & 537.6 & 704.5 \\
\hline & 1.98 & 0.016 & 0.05 & $\mathrm{RG}(\mathrm{SLy} 4)$ & 0.657 & $1.422 \cdot 10^{-8}$ & 33.62 & -2.997 & 14.20 & 901.8 & 923.0 \\
\hline
\end{tabular}

Table 1. Effective masses, interaction potentials and fractions of protons $x_{p}$ and neutrons $x_{n}$ under the thermodynamic conditions for which the opacities are shown in Figs. 2 and 3. For each given time, the first line corresponds to the smallest radius and the last one to the largest radius. $\Delta U=m_{n}^{*}-m_{p}^{*}+U_{n}-U_{p}-\left(m_{n}-m_{p}\right)$ is the shift in reaction threshold due to mean field effects, see Oertel et al. (2020).

considered as a guideline when applied to the entire temperature, electron fraction and density domain needed for simulating PNS cooling. The main physical effect should, however, be covered: as mentioned above, in regions where direct Urca reactions are allowed, they give the dominant contribution to (anti-)neutrino opacities and the collisional broadening only marginally influences the opacities. The essential effect of the mUrca processes as implemented here is thus to increase opacities above/below thresholds for the direct processes.

As an example, we show opacities within the different approximation schemes in Figs. 2 (neutrino) and 3 (antineutrinos) employing the RG(SLy4) EoS. The thermodynamic conditions for each panel correspond thereby to different times and different radii inside the star as obtained from PNS pro- files with the fiducial simulation employing MF rates and the RG(SLy4) EoS including MLT, see Sec. 4. The values for temperature, baryon number density and electron fraction are listed in Table 1. In many cases, the antineutrino opacities exhibit a pronounced threshold, where the increase in the opacity due to the collisional broadening in this region is clearly visible.

\section{SIMULATION RESULTS}

All simulations presented in this section are using the procedure described in Section 2, and include -unless otherwise stated- convective effects modelled by MLT. We have first checked our results with respect to the choice of the EoS, which has already been discussed by several authors 

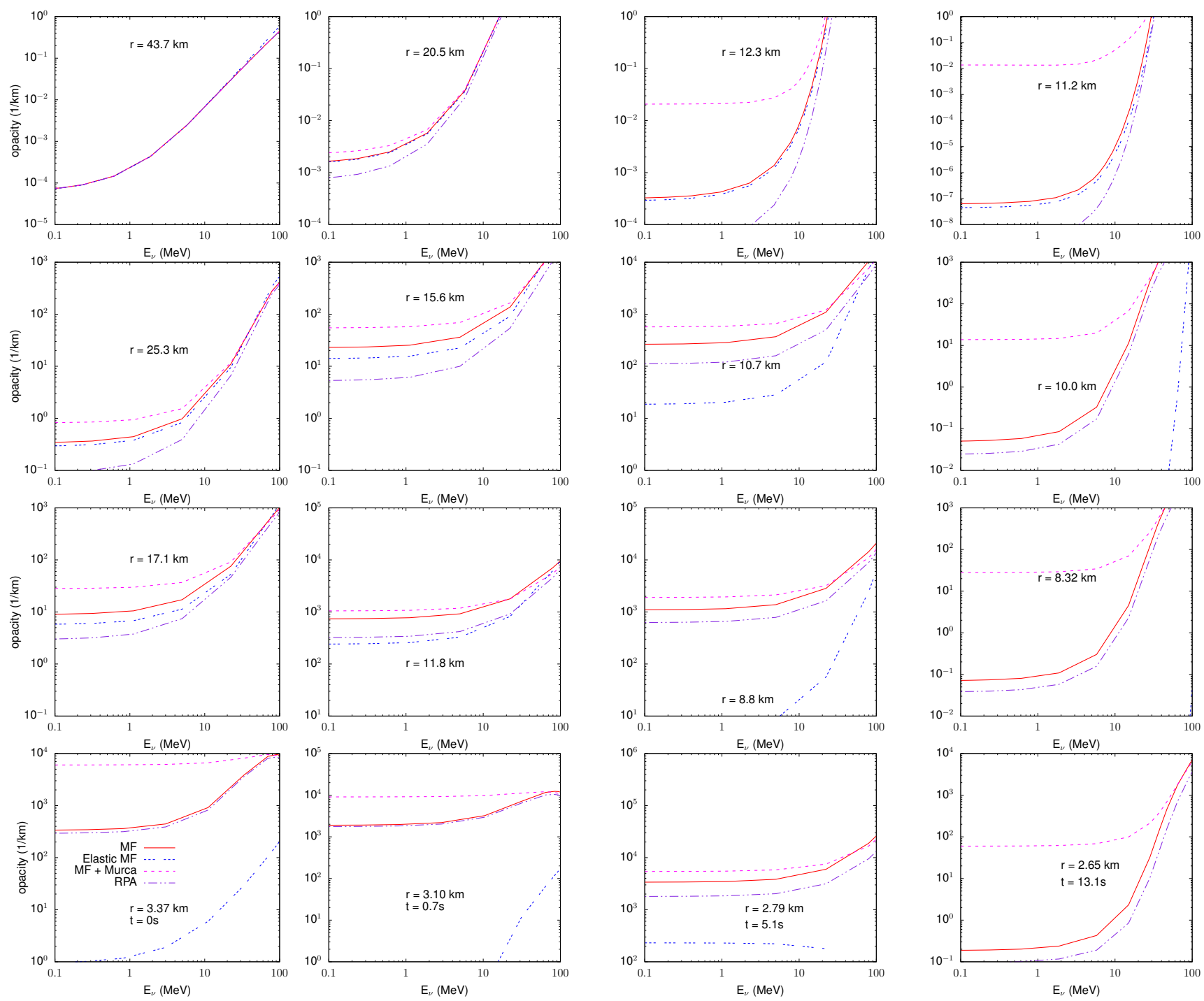

Figure 2. Neutrino $\left(\nu_{e}\right)$ opacities with the RG(SLy4) EoS using different approximation schemes for calculating the reaction rates. The different thermodynamic conditions, see Table 1 for the exact values, have been obtained from our fiducial simulation with the RG(SLy4) EoS and MF rates including MLT effects at different timesteps (increasing from left to right) and different locations (radius is increasing from bottom to top) in the star as indicated in the different panels. The uppermost panels correspond to conditions close to the surface of the star for that fiducial simulation.

(see e.g. Keil \& Janka 1995; Sumiyoshi et al. 1995; Pons et al. 1999). Among others, the influence of the symmetry energy on the PNS cooling timescale has been largely discussed (Sumiyoshi et al. 1995; Roberts et al. 2012b; Nakazato \& Suzuki 2019). We have performed simulations using the MF prescription and three different EoS models, RG(SLy4) (Gulminelli \& Raduta 2015), HS(DD2) (Hempel \& SchaffnerBielich 2010) and SRO(APR) (Schneider et al. 2019). All three of them have very similar symmetry energies at saturation, $J=32 ; 31.7 ; 32.6 \mathrm{MeV}$, respectively, and different slopes, $L=46 ; 55 ; 58 \mathrm{MeV}$.

We clearly confirm previous results in the literature and, in particular by Roberts et al. (2012b), according to which the time until the convection stops and the luminosity drops decreases with the symmetry energy slope of the equation of state. This indicates that the faster contraction of the PNS and in particular the convective effects outweigh the larger difference in neutrino and antineutrino opacities. Let us stress here, too, that although for the usually considered electron and proton capture reactions smaller symmetry energies indeed lead to larger difference in $\nu_{e}$ and $\overline{\nu_{e}}$ opacities (see e.g. the comparison for RG(SLy4) and HS(DD2) in Oertel et al. (2020)), this is no longer true for the neutron decay reaction and its inverse, whose importance for the low energy antineutrino opacity has been pointed out in Fischer et al. (2020a).

Finally, let us mention that in contrast to previous works we have used the same EoS, both in the PNS evolution simulations and for computing the initial model. As expected (see for instance the discussion in Pons et al. 1999), this has only little influence on the results for the long term PNS evolution. 

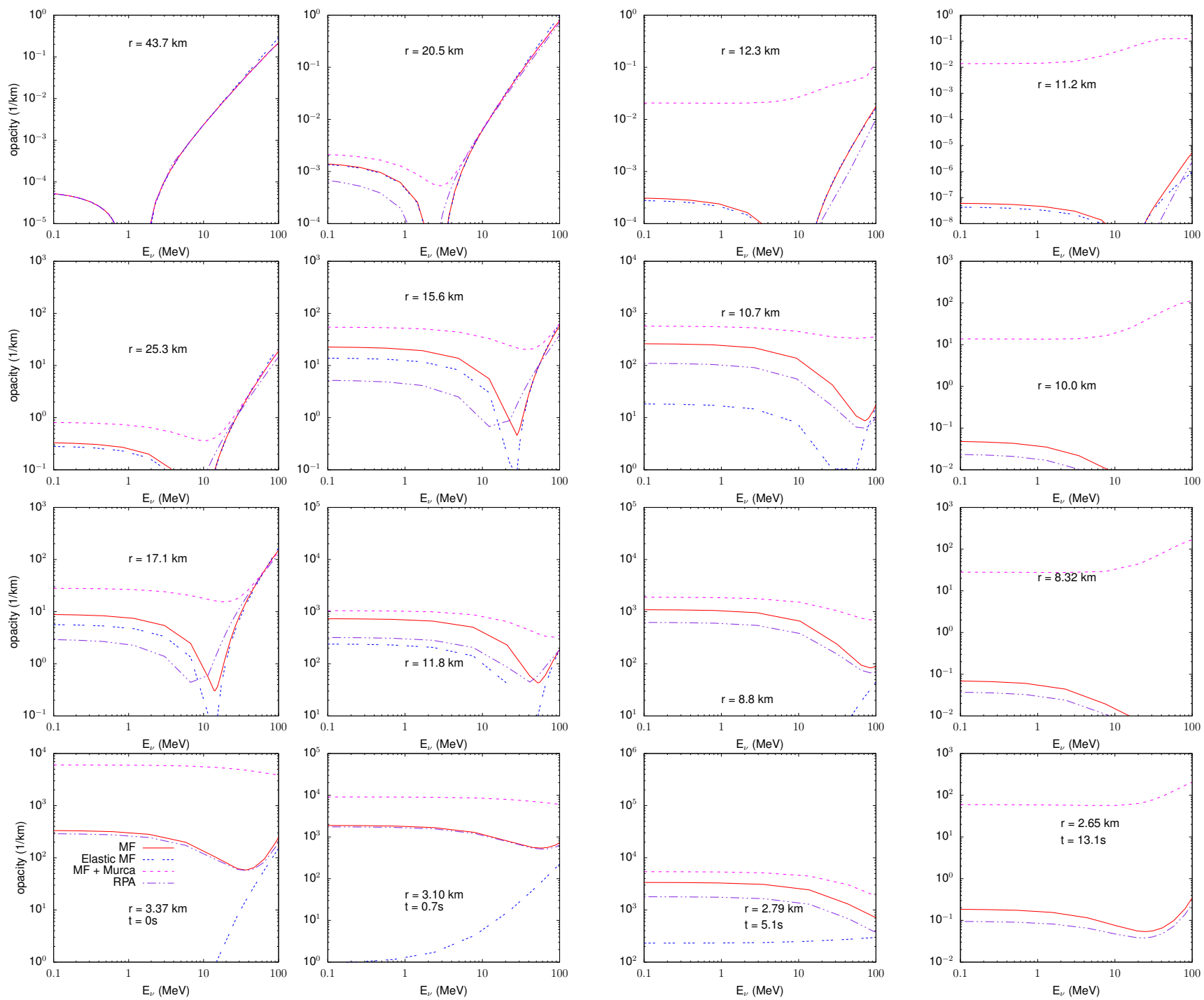

Figure 3. Same as Fig. 2 for antineutrino $\left(\overline{\nu_{e}}\right)$ opacities.

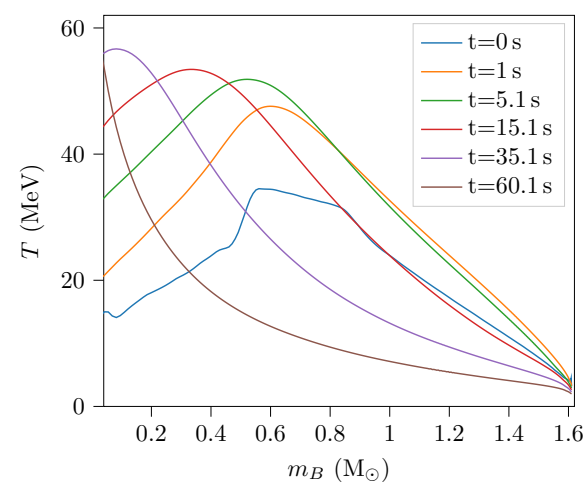

(a) Temperature $T$

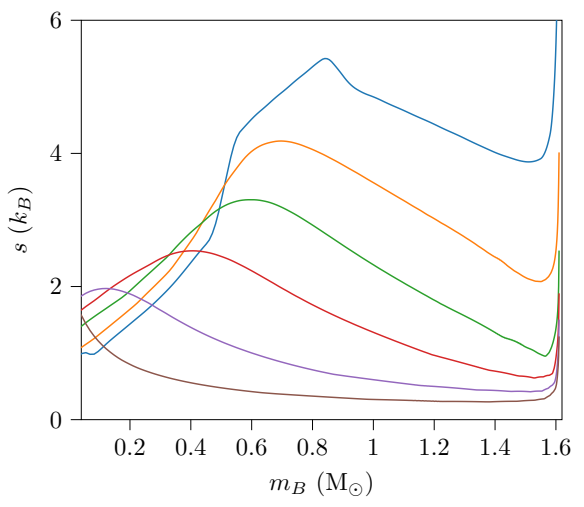

(b) Entropy per baryon $s$

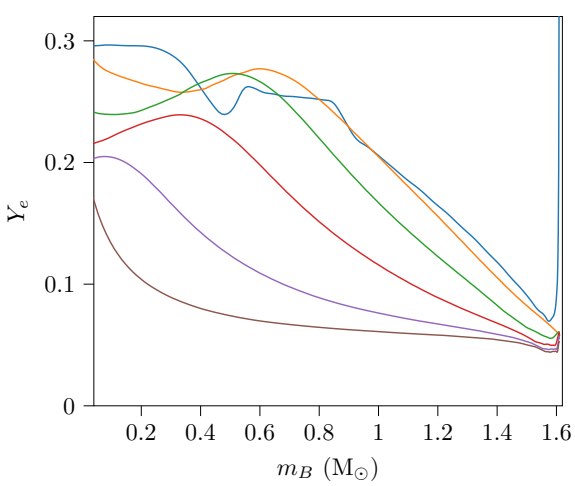

(c) Electron fraction $Y_{e}$

Figure 4. PNS internal structure at selected times in our fiducial simulation without mixing length theory. Various relevant thermodynamic quantities are plotted as functions of the enclosed baryon mass $m_{B}(r)=m_{N} a(r)$. 


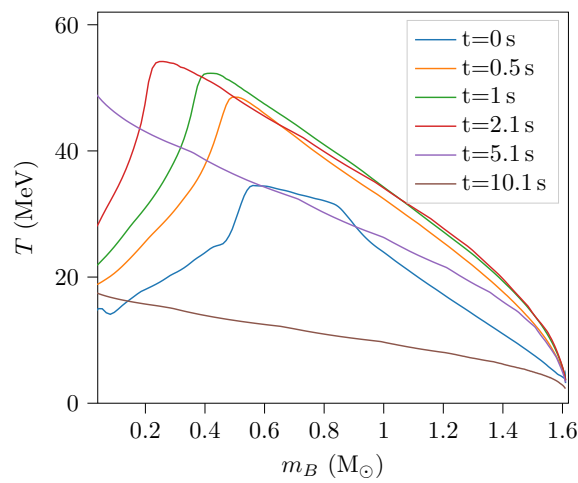

(a) Temperature $T$

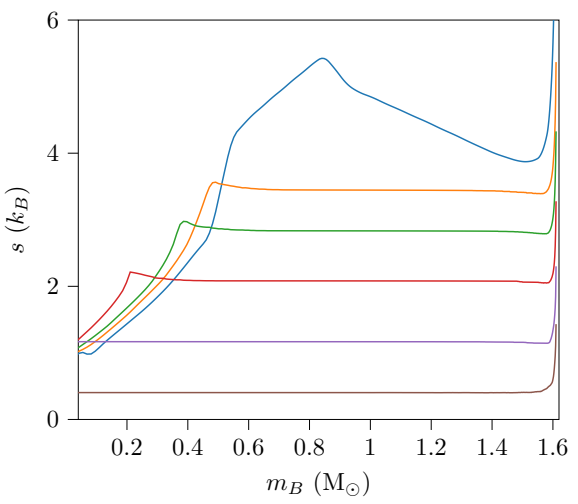

(b) Entropy per baryon $s$

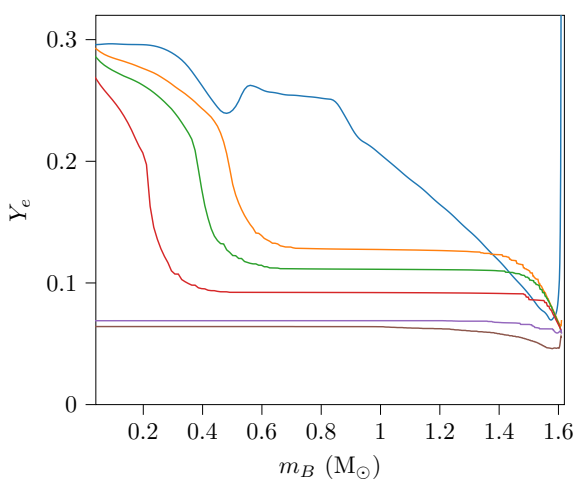

(c) Electron fraction $Y_{e}$

Figure 5. Same as Fig. 4 including convective effects via the mixing length theory.

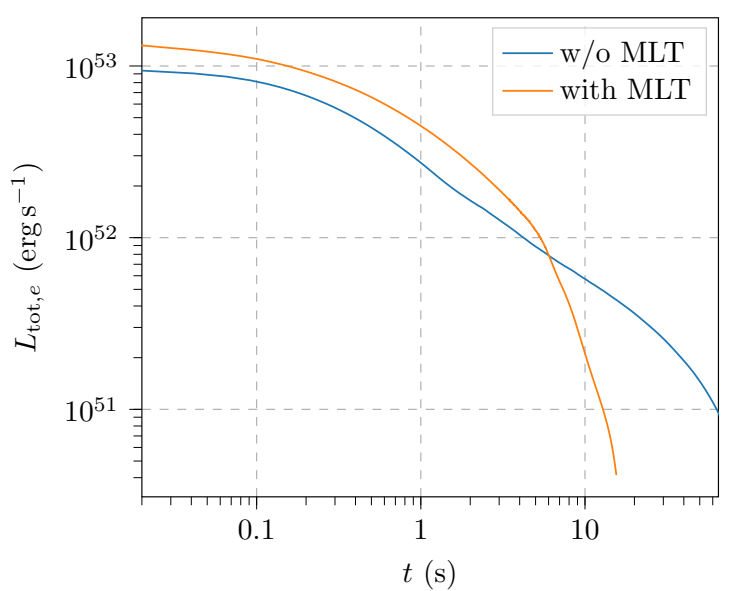

Figure 6. Total (sum of 6 flavors) neutrino energy luminosity as a function of time, in our fiducial simulation, with and without MLT.

\subsection{Influence of convection on proto-neutron star evolution}

In order to show the importance of convective effects during PNS evolution, we compare two simulations, with and without MLT. The EoS used for both the CCSN evolution and the quasi-static PNS modelling is RG(SLy4) (Gulminelli \& Raduta 2015) and the MF prescription for charged-current neutrino reaction rates has been employed, as described in Section 3 and in Oertel et al. (2020).

Fig. 4 shows the radial profiles of temperature $T$, entropy per baryon $s$ and electron fraction $Y_{e}$, as functions of the enclosed baryon mass $m_{B}$ at different times in our fiducial simulation which does not include convective effects. Fig. 5 shows the same profiles, but this time including convective effects via the MLT. The profiles at $t=0 \mathrm{~s}$ correspond to our initial data, which are taken at about $500 \mathrm{~ms}$ after bounce.

It is obvious that convection in the mantle of the PNS is extremely efficient: even by taking the same initial model as in our fiducial simulation (obtained with a spherically symmetric hydrodynamic simulation, thus without any convective effects) it takes less than about $100 \mathrm{~ms}$ to obtain a uniform profile of entropy per baryon $s$ and electron fraction $Y_{e}$ in the convectively unstable zone, which is then maintained in a state close to neutral buoyancy. These observations are qualitatively similar to what is obtained in full hydrodynamic studies, as for example in the Figs. 7 and 18 of Nagakura et al. (2020).

Another striking effect of including convective motions in the simulations is the difference in the evolution timescales: the non-convective model takes about four times longer than the convective one to fully deleptonize. Indeed, convective motions in the PNS are carrying leptons and heat from the inner boundary of the convective layer to its outer part, much closer to the neutrinospheres. As a consequence of the higher temperatures in the outer layers, the neutrino luminosity is about 1.5 times higher with MLT than without, as shown in Fig. 6. A sudden drop in the luminosity in observed after about $4-5 \mathrm{~s}$, when the star becomes homogeneous in entropy and electron fraction and the convective motions stop.

The emitted neutrino spectrum is also significantly modified by convection. Fig. 7 represents the mean energy of emitted neutrinos (as measured by a distant observer). We see that the mean energy of all neutrino flavors is globally enhanced during the early evolution with MLT, and then quickly drops when the star reaches neutral buoyancy and convection stops.

It should be stressed that we do not recover the results of Fig. 20 of Nagakura et al. (2020), where the authors observed that at early times the convective effects decrease the mean energy of emitted neutrinos. We do not believe our simulation to be accurate enough at that early epoch, as we neglected a lot of important physical ingredients such as the accretion onto the PNS, which is known to significantly alter the neutrino signal in particular by causing a time variation (see Nagakura et al. 2021). Instead, we focus on the long term evolution after the shock departure, for which our observations seem to be robust and confirm previous findings on the flattening of $s$ and $Y_{e}$ profiles as well as the luminosity, see e.g. Roberts et al. (2012b) and Roberts \& Reddy (2017).

In addition to the potentially observable drop in neutrino luminosities (Roberts et al. 2012b), the modifications in the neutrino spectra due to convective effects are quite important in the study of the neutrino driven wind (NDW). We estimate the electron fraction using the approximation developed in 


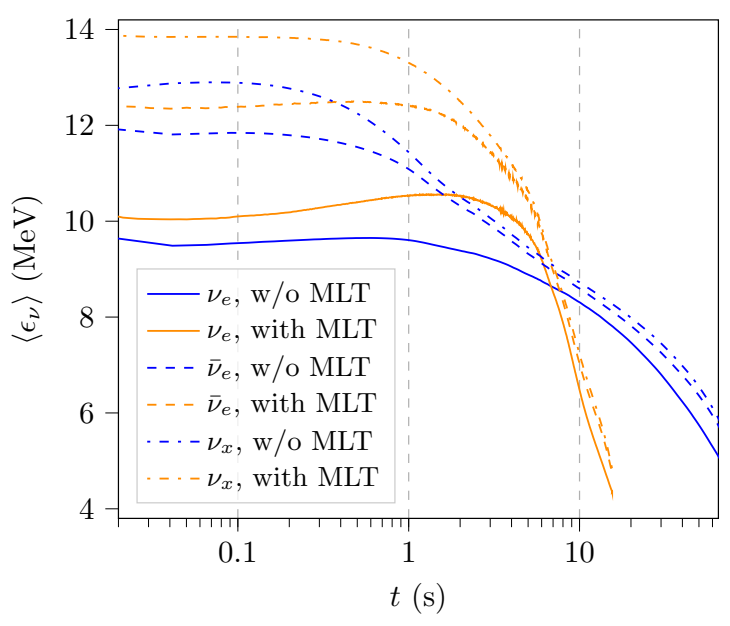

Figure 7. Mean energy of emitted neutrinos as a function of time, for each neutrino flavor, in our fiducial simulation, with and without MLT.

Qian \& Woosley (1996),

$Y_{e}^{\mathrm{NDW}}=\left[1+\frac{L_{\bar{\nu}_{e}, e}\left(\varepsilon_{\bar{\nu}_{e}}-2 \Delta+1.2 \Delta^{2} / \varepsilon_{\bar{\nu}_{e}}\right)}{L_{\nu_{e}, e}\left(\varepsilon_{\nu_{e}}+2 \Delta+1.2 \Delta^{2} / \varepsilon_{\nu_{e}}\right)}\right]^{-1}$

where $L_{\nu_{e}, e}$ and $L_{\bar{\nu}_{e}, e}$ are the electron neutrino and antineutrino energy luminosities, $\Delta=m_{n} c^{2}-m_{p} c^{2}$ is the neutronproton mass difference, and $\varepsilon_{\nu}$ is defined as $\varepsilon_{\nu}=\left\langle\epsilon_{\nu}^{2}\right\rangle /\left\langle\epsilon_{\nu}\right\rangle$ where $\epsilon_{\nu}$ is the energy of a given neutrino flavor $\nu$. This approximation considers only the processes $n+\nu_{e} \leftrightarrows p+e^{-}$ and $p+\bar{\nu}_{e} \leftrightarrows n+e^{+}$, in the elastic approximation neglecting the mass of the electron $m_{e}$ and the Pauli blocking effect of leptons on the final state.

It should be stressed that the formula (23) is obtained by considering that the NDW is composed of free neutrons and protons only. By doing so the so-called alpha effect is neglected, which results from the formation of $\alpha$ particles and can introduce significant changes in the electron fraction (see e.g. Meyer et al. 1998). But as our goal is only to estimate the global effect of modified neutrino spectra due to convective effects on the NDW, the formula (23) should nevertheless give us the global trend.

The evolution of the electron fraction in the NDW is represented in Fig. 8. We see that in our simulations convective effects push the composition of the NDW to higher electron fractions and that it is always proton-rich. The structure which can be observed in the simulation with MLT at about $4-5 \mathrm{~s}$ results from convection ceasing. Most recent CCSN simulations indicate a proton-rich NDW and under such conditions the most probable nucleosynthesis processes occurring in the wind are the weak r-process and the $\nu p$-process (see e.g. Arcones \& Thielemann 2013).

\subsection{Influence of neutrino interaction rates on the PNS evolution}

We study the influence of the various prescriptions for charged currents presented in Section 3 by performing simulations using either

- the elastic approximation with Mean Field corrections, without mUrca effects, denoted Elastic MF

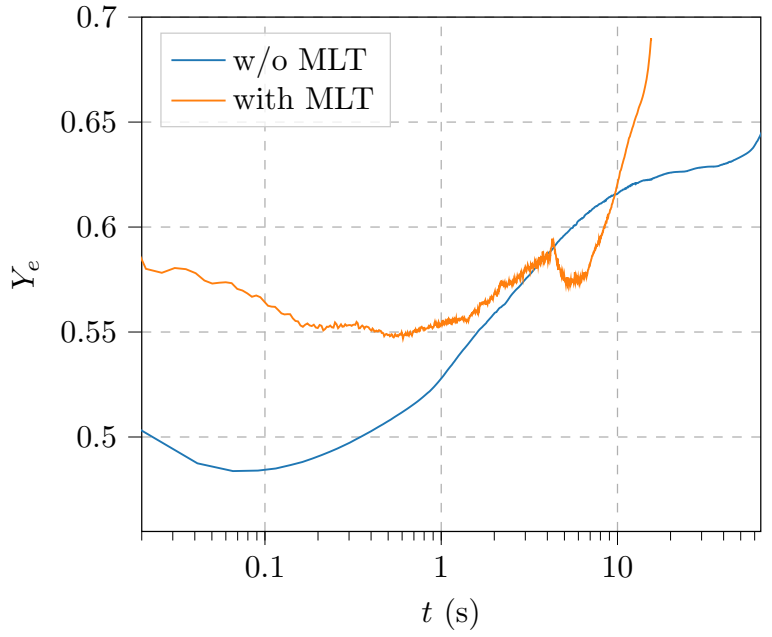

Figure 8. Electron fraction in the neutrino driven wind, computed using Eq. (23), in our fiducial simulation, with and without MLT.

- the Mean Field prescription without mUrca effects, denoted $\mathrm{MF}$

- the Mean Field prescription with mUrca effects, denoted $\mathrm{MF}+\mathrm{mUrca}$

- the RPA $t_{3}^{\prime}$ prescription without mUrca effects, denoted $\mathrm{RPA} t_{3}^{\prime}$

employing again the RG(SLy4) EoS (Gulminelli \& Raduta 2015).

We see little impact from these different prescriptions on the early PNS evolution during the convective phase. Some differences start to appear when convection stops and the luminosity starts to drop, as shown in Fig. 9, which shows the evolution of electron (anti-)neutrino luminosities as functions of time.

The differences caused by the elastic approximation start to be significant at this point, and the electron neutrino luminosity is much higher. This is explained by two factors: since the rates are lower the transport of energy and leptons in the PNS is more efficient, and the displacement of $\beta$-equilibrium towards more neutron rich matter leads to a more important deleptonization.

Regarding the effect of collisional broadening, the neutrino luminosities in simulations including mUrca are always lower than in those which do not. This can easily be understood. Since the opacities are globally enhanced by collisional broadening, the diffusion of heat and leptons inside the PNS is less efficient and the neutrinosphere is located at a lower temperature. This difference becomes more and more pronounced after about $10 \mathrm{~s}$ of evolution, when the temperature starts to drop, enhancing the differences between the prescriptions.

We can note here that, when comparing MF and MF with mUrca simulations, there are little differences in the energy luminosity (Fig. 9) and in the mean energy of emitted neutrinos (Fig. 10), whereas opacities are quite different, as seen from Figs. 2 and 3. This can be explained from the fact that the large differences in the rates occur in regions which are finally not so relevant for the neutrino emission and the overall evolution is dominated by other effects. In particular, the cooling of the central region is dominated by convective mixing whose dynamics are essentially independent of the 

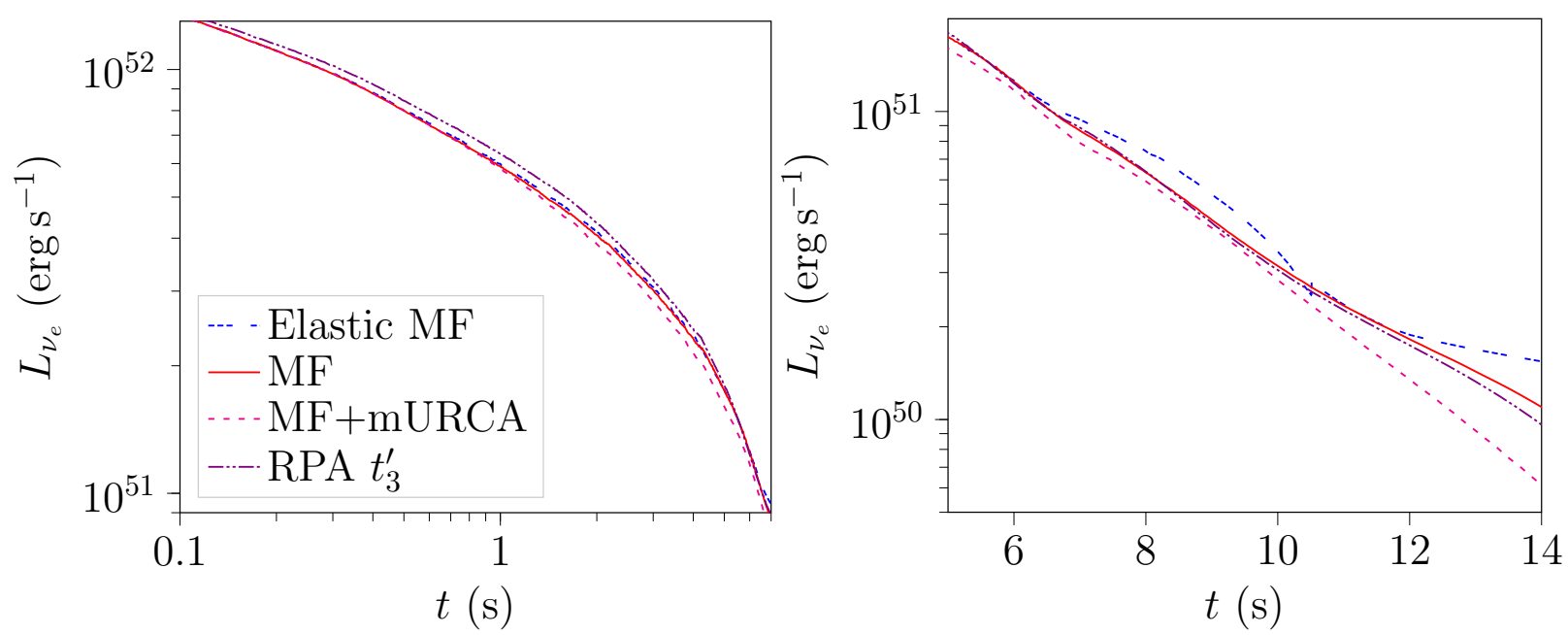

Figure 9. Energy luminosity of emitted electron neutrinos as function of time, for the four different prescriptions to compute charged current neutrino-nucleon interactions

charged currents. The exact location of the neutrinospheres is also not only determined by charged-current opacities but probably dominated by the scattering opacity which does not change between the different simulations. In addition, close to the neutrinospheres, relevant for neutrino emission, the differences in charged-current opacities are much smaller than in the centre or well above the neutrinospheres. We have checked this in our simulations, comparing the opacities for relevant thermodynamic conditions obtained from our fiducial run with MF rates in different regions of the PNS, see Figs 2 and 3. Close to the neutrinospheres, the difference in opacities with or without taking into account mUrca are not so large.

Finally, simulations which include RPA effects have a slightly higher luminosity during the first phase of the evolution. This can be understood because the reduced opacities due to nuclear correlations improves the diffusion of heat in the PNS. At later times this trend is inverted, since the model with RPA is colder and the neutrino mean free path becomes of the order of the size of the star. This behaviour is similar to the observations made in Fig. 5 of Roberts \& Reddy (2017), but some important differences can be noticed. Among others, in Roberts et al. (2012b); Roberts \& Reddy (2017) differences between RPA and MF start to appear only after convection has stopped, and become significant at late times, whereas our simulations show only small differences at late times. These discrepancies might have several origins. Differences coming from the initial model - we recall that we compute the initial model consistently with the same EoS and reaction rates as the simulation - could explain these different behaviours, as our RPA and MF results might differ at early times in contrast to those in Roberts \& Reddy (2017). As discussed in Oertel et al. (2020), the importance of RPA correlations is energy dependent and for some (anti)-neutrino energies almost no difference is observed with the MF opacities. Therefore, the inclusion of this energy dependence in our work in contrast to Roberts et al. (2012b); Roberts \& Reddy (2017) is another possible explanation for the discrepancies. We expect the average difference between RPA and MF to be reduced for the full rates compared with a grey fac- tor, which seems indeed to be the case. We also include the full inelasticity of neutrino-nucleon scattering, which leads to faster equilibration of neutrino spectra and thus reduces the differences between the different prescriptions for the charged current rates. The treatment of neutrino transport or the EoS certainly play a role, too.

Let us now have a look at the emitted neutrino spectrum. Fig. 10 represents the mean energy of emitted electron (anti)neutrinos (as measured by a distant observer). The findings are similar to those discussed above, the differences introduced by the elastic approximation are the most significant at late times, especially for electron neutrinos with enhanced mean energies. mUrca processes systematically lower the mean energy with increasing differences again appearing around $10 \mathrm{~s}$ of evolution. Globally, as for the luminosities, the inclusion of mUrca processes has a stronger impact on the mean energies than nuclear correlations included via RPA. The latter produces a stronger enhancement of neutrino mean energy and, to a lower extent, that of anti-neutrinos at early times. Here again, the trend is inverted at later times with mean energies of both electron neutrinos and antineutrinos slightly smaller with the RPA approach than in MF.

As far as the impact on the NDW is concerned, Fig. 11 (left panel) shows the evolution of the electron fraction in the NDW comparing the different prescriptions for computing charged current rates. It can be seen that the stronger enhancement within RPA at early times of the electron neutrino average energy, with respect to antineutrinos, (see Fig. 10) has some noticeable consequences on the electron fraction in the NDW, which is about $4 \%$ higher at early times than in MF. mUrca processes which essentially affect neutrinos and antineutrinos in the same way, show only little influence on the composition of the NDW at early times. The prediction of simulations including mUrca start to be different after about $8 \mathrm{~s}$, increasing the electron fraction. Note, however, that when determining $Y_{e}$ in the NDW with Eq. (23), we have used several approximations. In particular we have assumed only free nucleons, which should be considered with caution over such a long timescale.

Although in this paper we focus on the impact on charged 


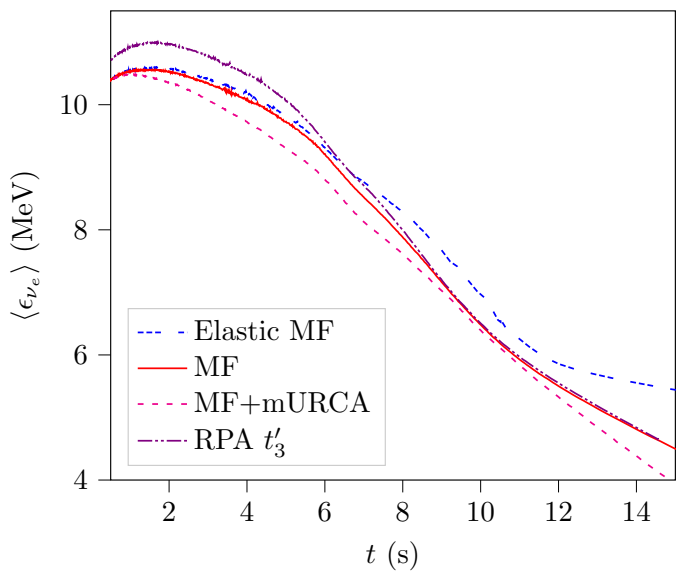

(a) Electron neutrino $\nu_{e}$

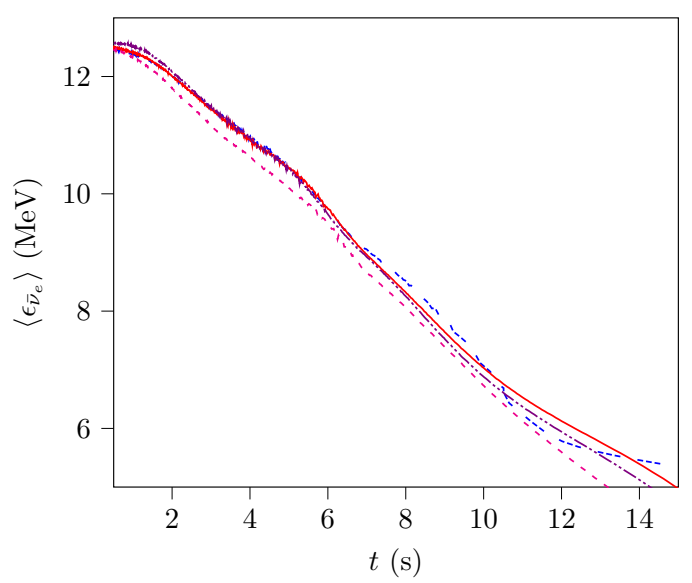

(b) Electron antineutrino $\bar{\nu}_{e}$

Figure 10. Mean energy of emitted electron (anti-)neutrinos as functions of time for the four different prescriptions for charged currents.

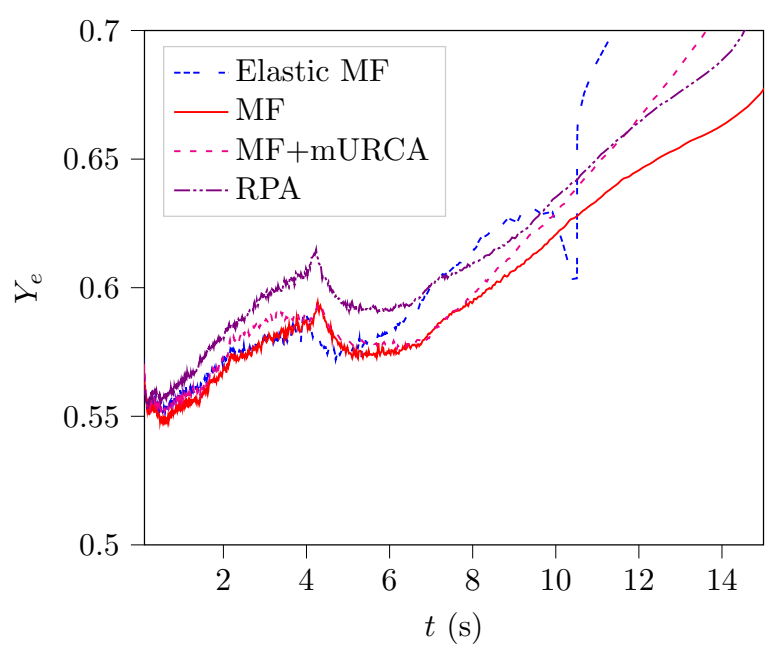

Figure 11. Electron fraction in the neutrino driven wind, computed with (23), as a function of time, for the four different prescriptions for charged currents. current rates, it should be noted that in most parts of the PNS, neutrino-nucleon scattering is the dominant source of opacity and different ways of computing the corresponding rates are expected as for the charged current rates to change the opacities and thus the PNS evolution. We will not perform here a detailed study, but as indication of the impact different scattering rates can have, we compare a simulation employing the elastic rates from Bruenn (1985) with our fiducial simulation using fully inelastic rates from Thompson et al. (2000), see appendix B. The overall evolution is again rather similar, see Fig. 12 where as an example we show the total neutrino energy luminosity and the electron fraction in the neutrino driven wind. At early times the elastic rates predict a slightly lower neutrino luminosity and a slightly more proton rich matter matter in the neutrino driven wind, but the evolution is again dominated by convection effects.

\section{SUMMARY AND DISCUSSION}

In this study we have performed simulations of the KelvinHelmholtz cooling phase of PNSs with a new code relying on the quasi-static approximation. Our code is modelling neutrino transport with the Fast Multigroup Transport (FMT) radiation scheme and includes convective effects within the mixing length theory (MLT).

We motivated the use of the mixing length theory by performing simulations with and without MLT, and conclude that simulations with convection yield results qualitatively different from simulations without. The PNS contracts much faster because of an efficient heat and lepton transport from inner regions to the neutrinospheres, the energy of emitted neutrinos is higher and the NDW is more proton-rich. As already discussed earlier, when convection stops there is a clear break in the neutrino luminosity, potentially observable.

In the continuation of our previous work on the influence of charged-current neutrino-nucleon interactions in corecollapse simulations (Oertel et al. 2020) we studied the influence of nuclear correlations and collisional broadening due to modified Urca processes in the evolution of PNSs. We recall that commonly used approximations in CCSN (the elastic approximation) and in NS cooling simulations (the Fermi surface approximation) are not compatible and that PNS evolution simulations should rely on charged current rates computed with full kinematics.

From our simulations we conclude that the addition of mUrca has a certain, though limited effect on the neutrino emission, by reducing both the mean energy and the luminosity of emitted electron (anti-)neutrinos. Moreover, the mUrca processes become dominant at low temperatures. This can be seen from Figs. 2 and 3, where we have taken the thermodynamic conditions from PNS profiles obtained with the fiducial simulation employing MF rates and the RG(SLy4) EoS at different times and at different radii inside the star. The highest radius at each given time is located close to the surface. It is obvious from that figure that at later times and closer to the surface, opacities from mUrca reactions dominate. A similar effect is observed for neutrinos. Thus although neutrino transparency has not been reached (the neutrino mean free path is still smaller than the size of the star) at the end of the simulation, we can confirm that mUrca processes dominate 

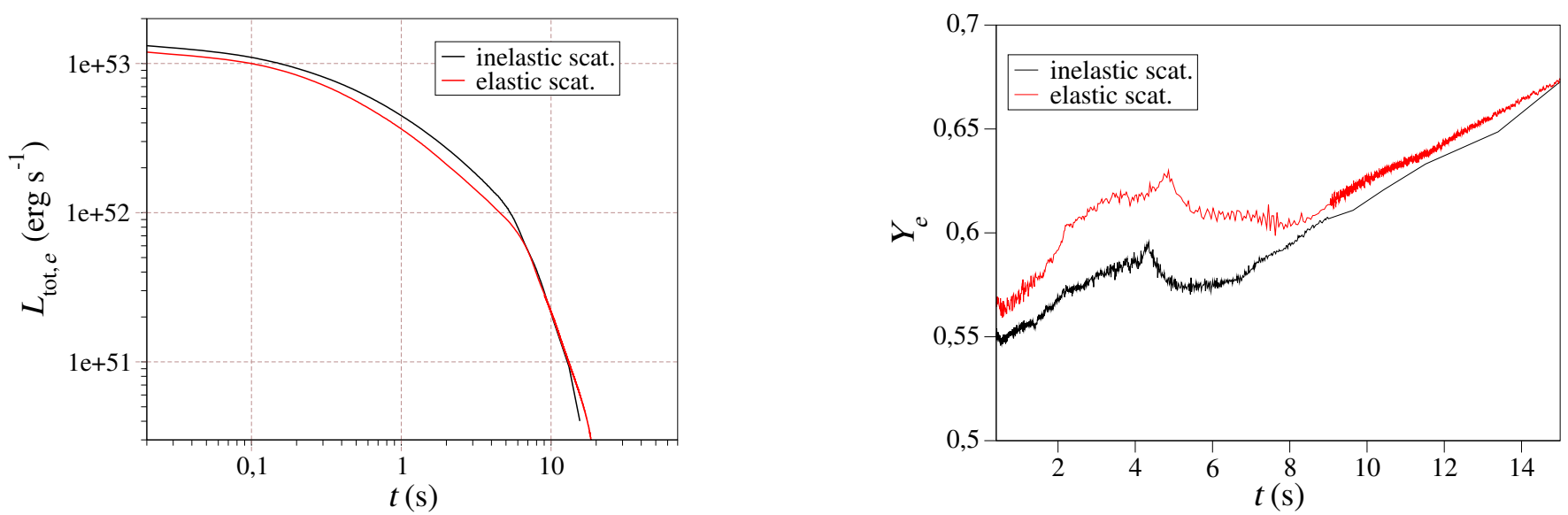

Figure 12. Total (6 flavors) neutrino energy luminosity (left) and electron fraction in the neutrino driven wind (right) computed with (23), as functions of time, comparing the elastic approximation (Bruenn 1985) for $\nu$ - $N$ scattering with the inelastic rates from (Thompson et al. 2000). The RG(SLy4) EoS and MF charged current rates have been employed.

the late evolution when direct processes become kinematically strongly suppressed.

The inclusion of nuclear correlations via RPA mostly has an effect at the beginning of the simulation, by enhancing the mean energy of emitted electron neutrinos, which indirectly increase the electron fraction in the NDW. At later times our simulations with RPA show little difference in the results, with respect to mean field rates. Thus although qualitatively in agreement with previous works (see e.g. Roberts \& Reddy 2017), there are some important quantitative discrepancies. As discussed in Sec. 4.2, there are several possible reasons for that: differences in the initial model, the energy dependence of the RPA rates included in our work, the faster equilibration of neutrino spectra due to inelastic scattering off nucleons, the neutrino treatment or the EoS. These different effects should be investigated further before any generic conclusion on the impact of nuclear correlations on PNS evolution and the resulting neutrino emission can be drawn.

Of course our study has several limitations. The simulations investigating the different prescriptions for charged current neutrino-nucleon interactions have been performed with only one EoS, thus we cannot exclude that other EoS show different effects. In addition, although we do not see much difference in the results from different progenitors, it should be mentioned that only a few have been used and that some particular progenitors might show different behaviour. The numerical method used could be improved, too. The neutrino transport scheme is an approximation to the full Boltzmann transport. It has the advantage of being computationally not very expensive and at the same time being more elaborate than standard equilibrium flux limited diffusion methods. In particular in the semi-transparent regime it behaves as well as some recently employed more sophisticated, but computationally more expensive methods (as e.g. variable Eddington transport methods in Roberts 2012). The transition between the CCSN evolution code with full-hydrodynamics and our quasi-static PNS evolution code induces a small discontinuity in some quantities, which, however, should only impact the very early evolution. As again shown here, convection plays a very important role for PNS evolution. The MLT scheme should govern the main qualitative features, but only a more detailed multi-dimensional study can fully account for convective effects. In addition, we have completely neglected the accretion process, therefore the first few hundreds of milliseconds in the evolution of our models should not be considered as reproducing accurately the conditions in CCSN.

We nevertheless demonstrated the feasibility of PNS studies including mixing length theory and state-of-the-art microphysics for the computation of neutrino interactions with a new computationally low-cost numerical algorithm. This kind of method allows to study a wide parameter space in a reasonable time and will certainly prove useful as uncertainties in PNSs evolution are still numerous.

\section{ACKNOWLEDGEMENTS}

We would like to thank B. Müller for providing us with the original FMT code and Tobias Fischer for discussions. The research leading to these results has received funding from the PICS07889 and the Observatoire de Paris through the action fédératrice "PhyFog". This work was granted access to the computing resources of MesoPSL financed by the Region Ile de France and the project Equip@Meso (reference ANR10-EQPX-29-01) of the programme Investissements d'Avenir supervised by the Agence Nationale pour la Recherche. The authors gratefully acknowledge the Italian Istituto Nazionale de Fisica Nucleare (INFN), the French Centre National de la Recherche Scientifique (CNRS) and the Netherlands Organization for Scientific Research for the construction and operation of the Virgo detector and the creation and support of the EGO consortium.

\section{DATA AVAILABILITY}

No new data were generated or analysed in support of this research.

\section{REFERENCES}

Akmal A., Pandharipande V., 1997, Phys. Rev. C, 56, 2261 
Akmal A., Pandharipande V., Ravenhall D., 1998, Phys. Rev. C, 58,1804

Alford M. G., Harris S. P., 2018, Phys. Rev. C, 98, 065806

Alford M. G., Haber A., Harris S. P., Zhang Z., 2021, arXiv eprints, p. arXiv:2108.03324

Arcones A., Thielemann F. K., 2013, Journal of Physics G Nuclear Physics, 40, 013201

Bacca S., Hally K., Liebendorfer M., Perego A., Pethick C. J., Schwenk A., 2012, Astrophys. J., 758, 34

Bollig R., Janka H. T., Lohs A., Martínez-Pinedo G., Horowitz C. J., Melson T., 2017, Phys. Rev. Lett., 119, 242702

Bruenn S. W., 1985, ApJS, 58, 771

Burrows A., Lattimer J. M., 1986, ApJ, 307, 178

Burrows A., Sawyer R. F., 1998, Phys. Rev., C58, 554

Burrows A., Sawyer R. F., 1999, Phys. Rev., C59, 510

Chabanat E., Bonche P., Haensel P., Meyer J., Schaeffer R., 1997, Nucl. Phys. A, 627, 710

Chernohorsky J., 1994, ApJ, 433, 247

Constantinou C., Muccioli B., Prakash M., Lattimer J. M., 2014, Phys. Rev. C, 89, 065802

Dimmelmeier H., Novak J., Font J. A., Ibáñez J. M., Müller E., 2005, Phys. Rev. D, 71, 064023

Epstein R. I., 1979, MNRAS, 188, 305

Fischer T., Whitehouse S. C., Mezzacappa A., Thielemann F. K., Liebendörfer M., 2010, A\&A, 517, A80

Fischer T., Guo G., Dzhioev A. A., Martínez-Pinedo G., Wu M.-R., Lohs A., Qian Y.-Z., 2020a, Phys. Rev. C, 101, 025804

Fischer T., Guo G., Martínez-Pinedo G., Liebendörfer M., Mezzacappa A., 2020b, Phys. Rev. D, 102, 123001

Friman B. L., Maxwell O. V., 1979, Astrophys. J., 232, 541

Gulminelli F., Raduta A. R., 2015, Phys. Rev. C, 92, 055803

Hannestad S., Raffelt G., 1998, Astrophys. J., 507, 339

Hempel M., Schaffner-Bielich J., 2010, Nucl. Phys. A, 837, 210

Horowitz C. J., Schwenk A., 2006, Phys. Lett., B642, 326

Horowitz C. J., Caballero O. L., Lin Z., O'Connor E., Schwenk A., 2017, Phys. Rev., C95, 025801

Hüdepohl L., Müller B., Janka H. T., Marek A., Raffelt G. G., 2010, Phys. Rev. Lett., 104, 251101

Iosif P., Stergioulas N., 2021, MNRAS, 503, 850

Keil W., Janka H. T., 1995, A\&A, 296, 145

Keil W., Janka H. T., Müller E., 1996, Astrophys. J. Lett., 473, L111

Li S. W., Roberts L. F., Beacom J. F., 2021, Phys. Rev. D, 103, 023016

Lykasov G. I., Pethick C. J., Schwenk A., 2008, Phys. Rev. C, 78, 045803

Margueron J., Navarro J., Blottiau P., 2004, Phys. Rev., C70, 028801

Martínez-Pinedo G., Fischer T., Lohs A., Huther L., 2012, Phys. Rev. Lett., 109, 251104

Meyer B. S., McLaughlin G. C., Fuller G. M., 1998, Phys. Rev. C, 58,3696

Mirizzi A., Tamborra I., Janka H. T., Saviano N., Scholberg K., Bollig R., Hüdepohl L., Chakraborty S., 2016, Nuovo Cimento Rivista Serie, 39, 1

Müller B., Janka H. T., 2015, MNRAS, 448, 2141

Nagakura H., Burrows A., Radice D., Vartanyan D., 2020, MNRAS, 492, 5764

Nagakura H., Burrows A., Vartanyan D., 2021, submitted

Nakazato K., Suzuki H., 2019, Astrophys. J., 878, 25

Nakazato K., Suzuki H., 2020, Astrophys. J., 891, 156

Nakazato K., et al., 2021, arXiv e-prints, p. arXiv:2108.03009

Navarro J., Hernandez E. S., Vautherin D., 1999, Phys. Rev., C60, 045801

Oertel M., Pascal A., Mancini M., Novak J., 2020, Phys. Rev. C, 102, 035802

Paschalidis V., Etienne Z. B., Shapiro S. L., 2012, Phys. Rev. D, 86,064032
Pons J. A., Reddy S., Prakash M., Lattimer J. M., Miralles J. A., 1999, ApJ, 513, 780

Prakash M., Lattimer J. M., Pons J. A., Steiner A. W., Reddy S., 2001, in Blaschke D., Glendenning N. K., Sedrakian A., eds, , Vol. 578, Physics of Neutron Star Interiors. Springer, p. 364

Qian Y. Z., Woosley S. E., 1996, ApJ, 471, 331

Reddy S., Prakash M., Lattimer J. M., 1998, Phys. Rev. D, 58, 013009

Reddy S., Prakash M., Lattimer J. M., Pons J. A., 1999, Phys. Rev., C59, 2888

Roberts L. F., 2012, ApJ, 755, 126

Roberts L. F., Reddy S., 2017, in Alsabti A. W., Murdin P., eds, , Handbook of Supernovae. Springer, p. 1605, doi:10.1007/9783-319-21846-5 5

Roberts L. F., Reddy S., Shen G., 2012a, Phys. Rev. C, 86, 065803 Roberts L. F., Shen G., Cirigliano V., Pons J. A., Reddy S., Woosley S. E., 2012b, Phys. Rev. Lett., 108, 061103

Schneider A., Constantinou C., Muccioli B., Prakash M., 2019, Phys. Rev. C, 100, 025803

Sumiyoshi K., Suzuki H., Toki H., 1995, A\&A, 303, 475

Suwa Y., 2014, PASJ, 66, L1

Thompson T. A., Burrows A., Horvath J. E., 2000, Phys. Rev. C, 62, 035802

Typel S., Röpke G., Klähn T., Blaschke D., Wolter H. H., 2010, Phys. Rev. C, 81, 015803

Typel S., Oertel M., Klähn T., 2015, Physics of Particles and Nuclei, 46, 633

Woosley S. E., Heger A., Weaver T. A., 2002, Reviews of Modern Physics, 74, 1015

Yakovlev D. G., Kaminker A. D., Gnedin O. Y., Haensel P., 2001, Phys. Rep., 354, 1

Yueh W. R., Buchler J. R., 1977, ApJ, 217, 565

\section{APPENDIX A: FAST MULTIGROUP TRANSPORT}

In a spherically-symmetric spacetime described by the metric (1), the stationary Boltzmann equation can be written as

$$
\begin{array}{r}
\frac{\mu}{\psi} \frac{\partial f}{\partial r}+\frac{\mu \epsilon}{\psi}\left(\partial_{r} \ln \alpha\right) \frac{\partial f}{\partial \epsilon} \\
+\left(\frac{1}{r}-\left(\partial_{r} \ln \alpha\right)\right) \frac{1-\mu^{2}}{\psi} \frac{\partial f}{\partial \mu}=\mathcal{B}[f]
\end{array}
$$

where $f(r, \epsilon, \mu)$ is the distribution function, $\epsilon=p^{0}$ is the energy of neutrinos and $\mu=\cos \Theta=p^{r} / p^{0}$ is the cosine of the propagation angle. In the stationary case we can treat the redshift by a simple change of variable : we introduce the redshifted energy $\hat{\epsilon}=\alpha \epsilon$. For the function $f(r, \hat{\epsilon}, \mu)$ the Boltzmann equation now simply becomes

$\frac{\mu}{\psi} \frac{\partial f}{\partial r}+\left(\frac{1}{r}-\left(\partial_{r} \ln \alpha\right)\right) \frac{1-\mu^{2}}{\psi} \frac{\partial f}{\partial \mu}=\mathcal{B}[f]$

Let us now introduce the three first angular moments of the distribution function : $J=\frac{1}{2} \int f d \mu, H=\frac{1}{2} \int f \mu d \mu$ and $K=$ $\frac{1}{2} \int f \mu^{2} d \mu$ and write the collision integral as $\mathcal{B}[f]=j-\chi f$, which is possible if we consider only isotropic processes (see appendix B). The first two moment equations are then

$$
\begin{aligned}
\frac{\alpha^{2}}{r^{2} \psi} \frac{\partial}{\partial r}\left(\frac{r^{2}}{\alpha^{2}} H\right) & =j-\chi J \\
\frac{1}{\psi} \frac{\partial K}{\partial r}+\left(\frac{1}{r}-\left(\partial_{r} \ln \alpha\right)\right) \frac{3 K-J}{\psi} & =-\chi H
\end{aligned}
$$

The solution of this system is computed by using the fast 
neutrinos transport scheme by Müller \& Janka (2015). In the high optical depth area the solution is obtained using a twostream approximation,

$$
\frac{1}{\psi} \frac{\partial f_{\text {out }}}{\partial r}=j-\chi f_{\text {out }}-\frac{1}{\psi} \frac{\partial f_{\text {in }}}{\partial r} \quad=j-\chi f_{\text {in }} .
$$

It should be stressed that these two equations are usually coupled because the coefficients $j$ and $\chi$ depend of the neutrino distribution function, though in our case we use the neutrino distribution of the previous timestep to compute them (see appendix B). The flux factor $h=H / J$ is obtained by assuming a continuous distribution $f(\epsilon, \mu) \propto \mathrm{e}^{a \mu}$

$h=1+\frac{2 f_{\text {in }} / f_{\text {out }}}{1-f_{\text {in }} / f_{\text {out }}}+\frac{2}{\ln \left(f_{\text {in }} / f_{\text {out }}\right)}$

and we can then solve the flux-divergence equation A2.

The solution at low optical depth is obtained with a twomoment closure:

$k(h)=\frac{K}{J}=\frac{1-2 h+4 h^{2}}{3}$

which can be used to transform the equation A3 into an ordinary differential equation for the flux factor

$$
\begin{array}{r}
\frac{d h}{d r}=\frac{1}{k-h k^{\prime}(h)}\left\{\left(\frac{1}{r}-\partial_{r} \ln \alpha\right)(k-1) h-\right. \\
\left.\psi\left(\chi h^{2}-k \frac{j}{J}+k \chi\right)\right\} .
\end{array}
$$

These two solutions are matched at the point $h=0.51$, to avoid the singular point $h=0.5$.

Finally, the neutrino fluxes used in equations (12) and (13) are computed as

$$
\begin{aligned}
& F_{\nu, n}(r)=\frac{4 \pi c}{(h c)^{3}} \int H(r, \hat{\epsilon}) \frac{\hat{\epsilon}^{2} d \hat{\epsilon}}{\alpha^{3}} \\
& F_{\nu, e}(r)=\frac{4 \pi c}{(h c)^{3}} \int H(r, \hat{\epsilon}) \frac{\hat{\epsilon}^{3} d \hat{\epsilon}}{\alpha^{4}} .
\end{aligned}
$$

\section{APPENDIX B: NEUTRINO COLLISION INTEGRAL}

We limit ourselves to isotropic scattering and pair production kernels, which allows us to recast the collision integral to the form

$\mathcal{B}[f]=j_{\mathrm{eff}}-\chi_{\mathrm{eff}} f$,

where the coefficients $j_{\text {eff }}$ and $\chi_{\text {eff }}$ depend on the distribution function $f$. These effective coefficients are computed using the value of $f$ from the previous timestep. This approximation allows us to use the FMT algorithm while having very little influence on the result. The fact that interaction kernels are non-isotropic is usually taken into account by including their first Legendre moments, but this is impossible within the FMT. These anisotropies are expected to have only an influence in the semi-transparent regime, and should not change our results qualitatively.

\section{B1 Scattering integral}

The dominant processes for scattering of neutrinos in dense nuclear matter are scattering off free nucleons $\nu+N \leftrightarrows \nu+N$, and on a less important level the scattering off free electrons/positrons $\nu+e^{ \pm} \leftrightarrows \nu+e^{ \pm}$. The scattering off nucleons is treated as if the nucleons were an ideal gas (see e.g. Thompson et al. 2000) with full inelasticity, and electrons/positrons are treated as a relativistic ideal gas (see Yueh \& Buchler 1977; Chernohorsky 1994).

The scattering integral can be written as

$$
\begin{array}{r}
\mathcal{B}_{S}[f]=\frac{1}{2} \int\left(\epsilon^{\prime}\right)^{2} d \epsilon^{\prime} d \mu^{\prime}\left\{R_{0}^{\text {in }}\left(\epsilon, \epsilon^{\prime}\right) f\left(\epsilon^{\prime}, \mu^{\prime}\right)[1-f(\epsilon, \mu)]\right. \\
\left.-R_{0}^{\text {out }}\left(\epsilon, \epsilon^{\prime}\right) f(\epsilon, \mu)\left[1-f\left(\epsilon^{\prime}, \mu^{\prime}\right)\right]\right\}
\end{array}
$$

where $R_{0}^{\text {in }}$ and $R_{0}^{\text {out }}$ are the zeroth Legendre moment of the ingoing and outgoing scattering kernels:

$R_{0}^{\text {in } / \text { out }}\left(\epsilon, \epsilon^{\prime}\right)=\int_{0}^{\pi} R^{\text {in } / \text { out }}\left(\epsilon, \epsilon^{\prime}, \cos \Theta\right) \sin \Theta d \Theta$.

They fulfil the in/out symmetry $R_{0}^{\text {in }}\left(\epsilon, \epsilon^{\prime}\right)=R_{0}^{\text {out }}\left(\epsilon^{\prime}, \epsilon\right)$, as well as the detailed balance condition $R_{0}^{\text {in }}\left(\epsilon, \epsilon^{\prime}\right)=$ $\mathrm{e}^{\left(\epsilon^{\prime}-\epsilon\right) /\left(k_{B} T\right)} R_{0}^{\text {out }}\left(\epsilon, \epsilon^{\prime}\right)$.

The effective transport coefficients can then be written as

$$
\begin{aligned}
j_{\mathrm{eff}, S}(\epsilon) & =\frac{1}{2} \int\left(\epsilon^{\prime}\right)^{2} d \epsilon^{\prime} R_{0}^{i n}\left(\epsilon, \epsilon^{\prime}\right) J\left(\epsilon^{\prime}\right) \\
\chi_{\mathrm{eff}, S}(\epsilon) & =j_{\mathrm{eff}, S}(\epsilon)+\frac{1}{2} \int\left(\epsilon^{\prime}\right)^{2} d \epsilon^{\prime} R_{0}^{\text {out }}\left(\epsilon, \epsilon^{\prime}\right)\left[1-J\left(\epsilon^{\prime}\right)\right]
\end{aligned}
$$

where $J(\epsilon)=\frac{1}{2} \int f(\epsilon, \mu) d \mu$ is the zeroth moment of the distribution function.

\section{B2 Pair production integral}

The dominant processes for neutrino pair production in dense nuclear matter are mainly the nucleon-nucleon bremsstrahlung $N+N \leftrightarrows N+N+\nu+\bar{\nu}$, and on a less important level the electron-positron annihilation $e^{-}+e^{+} \leftrightarrows \nu+\bar{\nu}$ Bremsstrahlung is treated with the analytic fit of Hannestad \& Raffelt (1998), and electron-positron annihilation is treated in the ultrarelativistic limit (see Bruenn 1985). The ultrarelativistic approximation is not justified when the PNS becomes cold enough such that the mean energy of neutrinos gets close to the electron mass $m_{e} c^{2}$. But as this process is subdominant a complete treatment should not generate important changes in the results. Instead we simply add a cutoff to emulate the $2 m_{e} c^{2}$ threshold.

The pair production integral depends upon the distribution function of the antineutrino $\bar{f}$ :

$$
\begin{array}{r}
\mathcal{B}_{P}[f, \bar{f}]=\frac{1}{2} \int\left(\epsilon^{\prime}\right)^{2} d \epsilon^{\prime} d \mu^{\prime}\left\{R_{0}^{p}\left(\epsilon, \epsilon^{\prime}\right)[1-f(\epsilon, \mu)]\left[1-\bar{f}\left(\epsilon^{\prime}, \mu^{\prime}\right)\right]\right. \\
\left.-R_{0}^{a}\left(\epsilon, \epsilon^{\prime}\right) f(\epsilon, \mu) \bar{f}\left(\epsilon^{\prime}, \mu^{\prime}\right)\right\}
\end{array}
$$

where $R_{0}^{p}$ and $R_{0}^{a}$ are the zeroth Legendre moment of the production and absorption kernels for pair production,

$R_{0}^{p / a}\left(\epsilon, \epsilon^{\prime}\right)=\int_{0}^{\pi} R^{p / a}\left(\epsilon, \epsilon^{\prime}, \cos \Theta\right) \sin \Theta d \Theta$.

They fulfil the detailed balance condition $R_{0}^{a}\left(\epsilon, \epsilon^{\prime}\right)=$ $\mathrm{e}^{\left(\epsilon+\epsilon^{\prime}\right) /\left(k_{B} T\right)} R_{0}^{p}\left(\epsilon, \epsilon^{\prime}\right)$. The effective transport coefficients can 
then be written as

$$
\begin{aligned}
j_{\mathrm{eff}, P}(\epsilon) & =\frac{1}{2} \int\left(\epsilon^{\prime}\right)^{2} d \epsilon^{\prime} R_{0}^{p}\left(\epsilon, \epsilon^{\prime}\right)\left[1-\bar{J}\left(\epsilon^{\prime}\right)\right] \\
\chi_{\mathrm{eff}, P}(\epsilon) & =j_{\mathrm{eff}, P}(\epsilon)+\frac{1}{2} \int\left(\epsilon^{\prime}\right)^{2} d \epsilon^{\prime} R_{0}^{a}\left(\epsilon, \epsilon^{\prime}\right) \bar{J}\left(\epsilon^{\prime}\right)
\end{aligned}
$$

where $\bar{J}(\epsilon)=\frac{1}{2} \int \bar{f}(\epsilon, \mu) d \mu$ is the zeroth moment of the antineutrino distribution function.

\section{APPENDIX C: PHENOMENOLOGICAL APPROACH TO INCLUDE MODIFIED URCA PROCESSES}

The neutrino emissivity from charged current processes can be written as (see e.g. Oertel et al. 2020, for details)

$$
\begin{aligned}
j\left(E_{\nu}\right)= & -\frac{G_{F}^{2} V_{u d}^{2}}{8} \int \frac{d^{3} k_{e}}{(2 \pi)^{3}} \frac{1}{E_{e} E_{\nu}} L^{\lambda \sigma} \operatorname{Im} \Pi_{\lambda \sigma}^{R}(q) \times \\
& f_{F}\left(E_{e}-\mu_{e}\right)\left(1+f_{B}\left(q_{0}\right)\right)+\text { positronic contribution }
\end{aligned}
$$

with a similar expression for antineutrinos and the mean free path related via detailed balance. $G_{F}$ denotes here the Fermi coupling constant and $V_{u d}$ the quark mixing matrix element entering the charged current processes with nucleons. $q=\left(E_{e}-E_{\nu}-\mu_{e}+\mu_{\nu}, \overrightarrow{k_{e}}-\overrightarrow{k_{\nu}}\right)$ with subscripts indicating electron or neutrino energy and chemical potential, respectively, and $f_{F / B}$ are fermionic or bosonic distributions functions. Assuming non-relativistic nucleons and neglecting the momentum dependence of the nucleonic form factors, the product of the lepton tensor $L^{\lambda \sigma}$ and the retarded polarisation $\Pi^{R}$ becomes

$$
\begin{aligned}
L^{\lambda \sigma} \Pi_{\lambda \sigma}^{R}=8 & \left(\Pi_{V}\left(2 E_{e} E_{\nu}-K_{e} \cdot K_{\nu}\right)\right. \\
& \left.+\Pi_{A}\left(2 E_{e} E_{\nu}+K_{e} \cdot K_{\nu}\right)\right),
\end{aligned}
$$

with a vector $\Pi_{V}$ and an axial part $\Pi_{A}$. In mean field approximation for the direct processes, we obtain

$\operatorname{Im} \Pi_{V}(q)=\operatorname{Im} \Pi_{A}(q)=2 \operatorname{Im} L(q)$,

with the well-known Lindhard function $L(q)$,

$L(q)=\lim _{\eta \rightarrow 0} \int \frac{d^{3} k}{(2 \pi)^{3}} \frac{f_{F}\left(\epsilon_{k}^{p}-\mu_{p}^{*}\right)-f_{F}\left(\epsilon_{k+q}^{n}-\mu_{n}^{*}\right)}{\tilde{q_{0}}+i \eta+\epsilon_{k}^{p}-\epsilon_{k+q}^{n}}$.

with $\tilde{q_{0}}=q_{0}+\mu_{n}^{*}-\mu_{p}^{*}$. The single particles energies are $\epsilon_{k}^{i}=\frac{\vec{k}^{2}}{2 m_{i}^{*}}+m_{i}^{*}$ with effective masses $m_{i}^{*}$, and $\mu_{n / p}^{*}$ denote proton and neutron effective chemical potentials. Values for the effective masses and chemical potentials for the conditions of Figs. 2 and 3 are listed in Table 1. The approach by Roberts et al. (2012a) to describe modified URCA processes consists in considering a finite lifetime $\tau$ for the quasiparticles entering the Lindhard function in the axial channel leading to

$$
\begin{aligned}
\Pi_{A}(q)=2 & \int \frac{d^{3} k}{(2 \pi)^{3}} \frac{f_{F}\left(\epsilon_{k}^{p}-\mu_{p}^{*}\right)-f_{F}\left(\epsilon_{k+q}^{n}-\mu_{n}^{*}\right)}{\tilde{q_{0}}+i \tau+\epsilon_{k}^{p}-\epsilon_{k+q}^{n}} \\
& \times\left(1-\frac{i}{\tau} \frac{1}{\epsilon_{k+q}^{n}-\epsilon_{k}^{p}}\right)
\end{aligned}
$$

The values for $\tau$ have been taken from Bacca et al. (2012).

This paper has been typeset from a $\mathrm{TE}_{\mathrm{E}} / \mathrm{LAT}_{\mathrm{E}} \mathrm{X}$ file prepared by the author. 